\title{
Theory of Near-Adiabatic Collisions. II. Scattering Coordinate Method
}

W. R. Thorson

John B. Delos

William \& Mary, jbdelos@wm.edu

Follow this and additional works at: https://scholarworks.wm.edu/aspubs

Part of the Physics Commons

\section{Recommended Citation}

Thorson, W. R. and Delos, John B., Theory of Near-Adiabatic Collisions. II. Scattering Coordinate Method (1978). Physical Review A, 18(1), 135-155.

https://doi.org/10.1103/PhysRevA.18.135

This Article is brought to you for free and open access by the Arts and Sciences at W\&M ScholarWorks. It has been accepted for inclusion in Arts \& Sciences Articles by an authorized administrator of W\&M ScholarWorks. For more information, please contact scholarworks@wm.edu. 


\title{
Theory of near-adiabatic collisions. II. Scattering coordinate method
}

\author{
W. R. Thorson \\ Chemistry Department, The University of Alberta, Edmonton, Alberta, Canada T6G 2G2
}

J. B. Delos

Physics Department, The College of William and Mary, Williamsburg, Virgina 23185

(Received 24 June 1977)

\begin{abstract}
A rigorously correct and fully quantum-mechanical theory of slow atomic collisions is presented, which removes the formal defects and spurious nonadiabatic couplings of perturbed-stationary-states theory, and arrives at coupled equations for the heavy-particle motion which are the same as those obtained in the preceding paper by the electron translation factor formulation. Here, however, the theory is formulated in terms of suitably defined scattering coordinates, and electron translation factors do not appear. A unified physical interpretation of both approaches can thereby be made, and smaller terms in the coupled equations, describing corrections of order $m / \mu$ to electronic binding energies and to the collision kinetic energy, are placed on a firmer footing. Particular attention is paid to the critical test case of isotopic systems such as $\mathrm{HD}^{+}$and it is shown how a correct theory of isotopic charge exchange can be formulated.
\end{abstract}

\section{INTRODUCTION}

Much of the motivation for this paper is provided by work described in the preceding paper. ${ }^{1}$ There we have shown how the incorporation of electron translation factors (ETF's) into the Born-Oppenheimer molecular basis functions modifies the coupled equations of slow collision theory and corrects the flaws which arise from the improperly formulated perturbed-stationary states (PSS) theory. Our derivation there gives the correct coupled equations to replace those of PSS theory, but is not quite satisfactory since it rests on a quantum transcription formula. We were seeking a fully quantum-mechanical description of slow collisions, but ETF's normally refer to a classical heavy-particle velocity. We obtained a quantum-mechanical description by replacing this velocity with the corresponding operator and then performing the transformation of the Hamiltonian generated by this operator.

In this paper we give a rigorous quantum-mechanical derivation of the coupled equations. This removes any doubt about their validity and their correct detailed form. Furthermore, it unifies and gives a simple physical interpretation to two different and previously unrelated approaches to the problem of slow collisions. We have already described the ETF approach. ${ }^{1}$ The second approach was originally conceived by Mittleman ${ }^{2}$ and his co-workers, who showed that the effects of ETF's can al'so be obtained by making use of a nonlinear coordinate transformation, which is so defined that the new coordinates become the appropriate atomic frame coordinates in each asymptotic channel configuration, while in the collision region they provide a coordinate system locally adapted to the position of the molecular electron. This approach is fully quantum-mechanical and in it no reference to ETF's ever occurs. Our derivation differs from Mittleman's in certain respects, and lends itself more readily to intuitive physical interpretation, since the results can be compared directly with those obtained via the more familiar ETF approach, as well as deduced in a systematic manner from general concepts of reaction coordinates.

In addition to these very general objectives, there is a quite specific problem which has been a direct stimulus for much of our work both here and in the previous paper, and we believe that some of our procedure and its apparently unnecessary attention to details will be made much clearer if we describe this problem and give a brief history of its solution. The problem provides the most critical test of the adequacy of the theory.

Consider the process of charge exchange in an "isotopic molecule" such as the $\mathrm{HD}^{+}$system,

$$
\mathrm{H}^{+}+\mathrm{D}(1 s) \rightarrow \mathrm{H}(1 s)+\mathrm{D}^{+} .
$$

This system is electrically symmetric and therefore the problem at first seems to be a simple case of resonant charge exchange. However, since the nuclear masses differ, the reduced mass of an electron on D is slightly larger than it is on $\mathrm{H}$ and reaction (1.1) as written is endothermic by $29.8 \mathrm{~cm}^{-1}(0.0037 \mathrm{eV})$. (In the near future, it may be possible to measure the total cross section for this reaction at energies down to threshold by the merging beams technique. ${ }^{3}$ )

Recently, Hunter and Kuriyan have made a thorough theoretical study of this reaction, using the PSS theory. ${ }^{4}$ But PSS theory for an isotopic molecule like $\mathrm{HD}^{+}$gives a very strange result. 
The molecular electronic states for $\mathrm{HD}^{+}$are parity eigenfunctions just like those for $\mathrm{H}_{2}{ }^{+}$or $\mathrm{D}_{2}{ }^{+}$, since the electronic Hamiltonian remains symmetric. The small isotopic differences in $\mathrm{D}$ and $\mathrm{H}$ binding energies do appear in PSS theory, but as (velocity-independent) first-order perturbations. However, even when these real effects are neglected, PSS theory still predicts that electronic states of opposite parity are linked by a velocity-dependent nonadiabatic coupling of dipolar form, given by

$$
(-i m / 2 \hbar) \lambda\left(\epsilon_{g}-\epsilon_{u}\right)\langle g|\overrightarrow{\mathbf{r}}| u\rangle\left[(-i \hbar / \mu) d \chi_{u}(R) / d R\right]
$$

(see the Appendix). Here $|g\rangle$ and $|u\rangle$ are gerade and ungerade electronic states, with energies $\epsilon_{g}(R)$, $\epsilon_{u}(R) ; \chi_{u}(R)$ is the wave function for heavy-particle motion, and $[(-i \hbar / \mu) d / d R]$ the radial velocity operator; $\lambda$ is the mass asymmetry parameter, $\lambda$ $=\left(M_{A}-M_{B}\right) /\left(M_{A}+M_{B}\right)\left(\right.$ for $\left.\mathrm{HD}^{+}, \lambda=\frac{1}{3}\right)$. Some careful thought will show that this coupling makes no physical sense:

(i) In the first place, couplings violating electronic parity in $\mathrm{HD}^{+}$should only arise from the very tiny shifts in electron binding energies and wave functions associated with the electron reduced mass differences. But the coupling (1.2) is very substantial, and because of its velocity dependence it clearly has no relation to these effects.

(ii) Furthermore we show in the Appendix the following paradox. Suppose we formulate the theory from the outset in terms of a classical trajectory theory; that is, we assume the nuclei move on a specified classical trajectory $\vec{R}(t)$ and we solve the resulting time-dependent Schrödinger equation for the electron system. It is clear that though the electronic Hamiltonian is time dependent, it is parity symmetric, and no $(\underline{g}, \underline{u})$ couplings can possibly occur (unless we introduce in addition the neglected isotope splittings as an ad hoc perturbation). On the other hand, if we first formulate the theory quantally, in the PSS framework, and then take the resulting coupled equations to the classical limit (i.e., the limit of large mass at fixed nuclear velocity, as defined by Riley $\left.{ }^{5}\right)$, then the velocity-dependent $(g, \underline{u})$ couplings in (1.2) remain. This paradox is only resolved by recognizing that Eq. (1.2) is a fictitious coupling, an artifact of PSS theory which cannot appear in a properly formulated theory.

In an early (unpublished) analyis of the effects of electron translation factors in slow collisions, we (incorrectly) wrote the ETF in the form

$$
\exp \{(i m / 2 \hbar)[f(\overrightarrow{\mathbf{r}} ; \overrightarrow{\mathbf{R}})+\lambda] \overrightarrow{\mathrm{v}} \cdot \overrightarrow{\mathbf{r}}\}
$$

rather than in the forms we now know to be correct, such as

$$
\exp \left\{(i m / 2 \hbar)[f(\overrightarrow{\mathrm{r}} ; \overrightarrow{\mathrm{R}})+\lambda] \overrightarrow{\mathrm{v}} \cdot\left[\overrightarrow{\mathrm{r}}-(f+\lambda) \frac{1}{4} \overrightarrow{\mathrm{R}}\right]\right\}
$$

or

$$
\begin{aligned}
\exp ((i m / 2 \hbar) \overrightarrow{\mathrm{V}}:\{ & f(\overrightarrow{\mathrm{r}} ; \overrightarrow{\mathrm{R}})+\lambda]\left(\overrightarrow{\mathrm{r}}-\frac{1}{2} \lambda \overrightarrow{\mathrm{R}}\right) \\
& \left.\left.-\frac{1}{4}\left(1-\lambda^{2}\right) \frac{1}{4} \overrightarrow{\mathrm{R}}\right\}\right) .
\end{aligned}
$$

When we developed the ETF theory using (1.3), we found that the resulting correction matrix elements $\overrightarrow{\mathrm{A}}(R)$ exactly cancelled the $(\underline{g}, \underline{u})$ couplings (1.2) of PSS theory, but to our dismay they replaced it with a new and equally paradoxical $(\underline{g}, \underline{u})$ coupling

$$
\begin{aligned}
(i m / 2 \hbar)\left(\epsilon_{g}\right. & \left.-\epsilon_{u}\right)\left(\frac{1}{2} \lambda \overrightarrow{\mathrm{R}}\right) \\
& \times\langle g|f(\overrightarrow{\mathrm{r}} ; \overrightarrow{\mathrm{R}})| u\rangle\left[(-i \hbar / \mu) d \chi_{u} / d R\right] .
\end{aligned}
$$

When, however, by careful reconsideration of details previously overlooked, we obtained the correct forms for ETF's, (1.4), all fictitious velocity-dependent $(\underline{g}-u)$ couplings were finally eliminated and we arrived at the ETF formulation of the preceding paper. ${ }^{1}$

However, the following problem remains: though the simplified coupled equations (3.13) of Ref. 1 give a consistent and correct account of nearly all collision processes, they do not provide an adequate formulation of the specific problem of isotopic molecules. The isotopic binding energy effects relevant for that problem are contained in the additional terms (in $\vec{A}$ and $\Delta$ ) appearing in Eqs. (3.7) of Ref. 1. Equations (3.7) are indeed correct, as we will prove here, but the form of these smaller terms is not convincingly established in the ETF formulation. In particular, different choices in the form and manner of introduction of the transcription formula lead to different results for these terms. The matrix $\Delta$ is especially difficult to interpret within the ETF framework. Thus there is a need for a completely rigorous derivation which is quantum mechanical from the outset.

Such a derivation is presented in this paper, using a somewhat different form of the coordinate transformation method originally conceived by Mittleman. ${ }^{2}$ Mittleman's specific results do not give quite the correct solution; when applied to the $\mathrm{HD}^{+}$problem, they yield the fictitious $(\underline{g}, \underline{u})$ coupling (1.5), ${ }^{6}$ a result we now know is incorrect. Our derivation also differs significantly in its treatment of the electronic states, and our starting point is somewhat more systematic. The essential idea is the use of the mass-scaled coordinates which are familiar in chemical reactive scattering theory. In these coordinates the collision is described as the motion of a single particle on a six-dimensional surface (for a single electron and two heavy particles $A, B$ ). If we 
express both molecular and atomic coordinate systems in these coordinates, and then seek a variable coordinate system which is continuously adapted to the electron's location, a simple definition of the scattering coordinate is suggested immediately and the whole theory then falls out correctly in a natural way.

In the ETF formulation, the switching function $f(\overrightarrow{\mathrm{r}} ; \overrightarrow{\mathrm{R}})$ appears in the ET F [Eqs. (1.4)]; in this method, it shows up in the definition of the new "variable" coordinate system. Just as before, the derivation provides no scheme for the unique determination of the switching function (apart from the asymptotic boundary conditions). Section IV of Ref. 1 discusses questions raised by this lack of uniqueness.

As in Ref. 1, we consider only the prototype case of a single electron. It is possible to extend the treatment to a many-electron system in a reasonably direct way, but several complications and further questions arise, and if we attempted to consider them here as well they could obscure the main features of the problem.

\section{SYSTEM DESCRIPTION}

\section{A. Coordinate systems}

We consider a system of one electron (rest mass $m_{0}$ ) and two heavy particles $A, B$ (masses $M_{A}$, $M_{B}$; without loss of generality we take $M_{A} \geqslant M_{B}$ ). Relative to a fixed origin their vector coordinates are $\overrightarrow{\mathbf{r}}^{0}, \overrightarrow{\mathrm{R}}_{A}^{0}, \overrightarrow{\mathrm{R}}_{B}^{0}$. The center-of-mass motion is removed in the usual way, and the remaining rela-
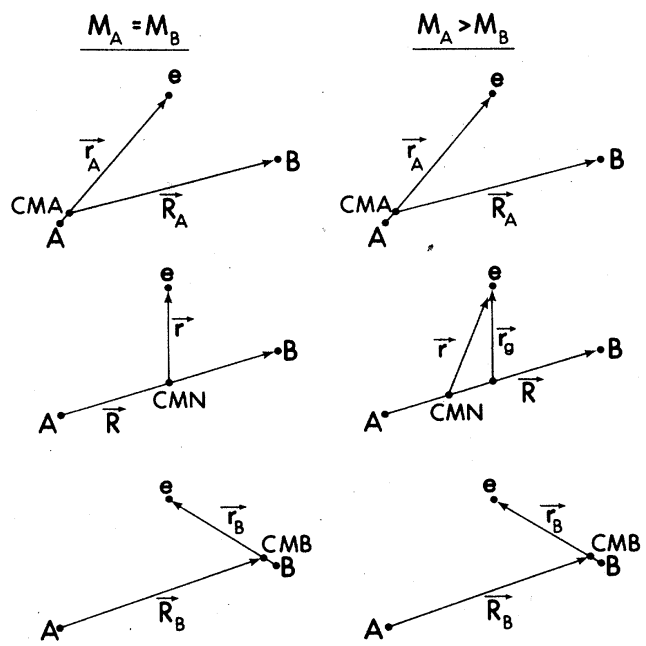

FIG. 1. Three Jacobi coordinate systems for relative coordinates of two heavy particles $A, B$ and an electron $e$, for equal masses, $M_{A}=M_{B}$, and for unequal masses, $M_{A}$ $>M_{B}$. CMN denotes center of mass of $A, B$.
TABLE I. Relations of coordinates.

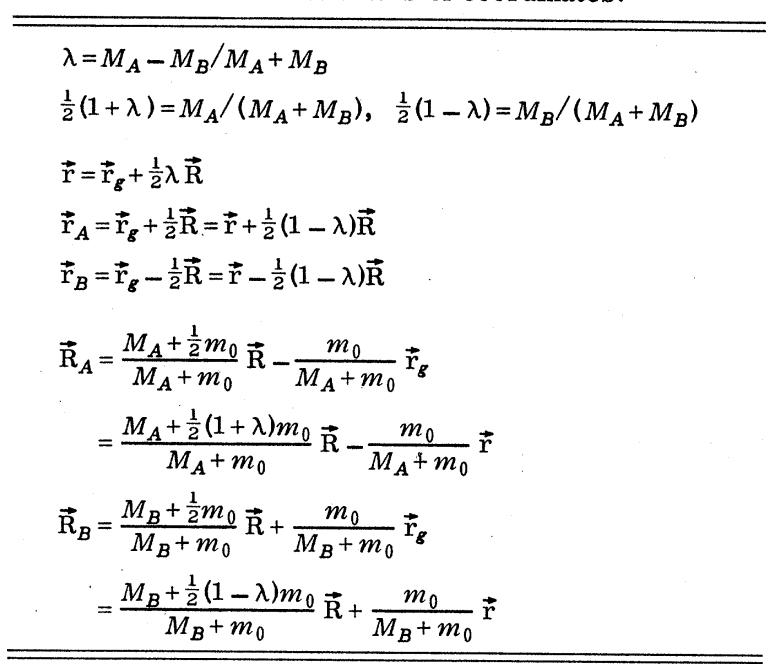

tive coordinates are defined so that the kinetic energy contains no "cross terms" like $\vec{\nabla}_{r} \cdot \vec{\nabla}_{R}$. The three standard (Jacobi) coordinate systems ${ }^{7}$ that satisfy this requirement are shown in Fig. 1. In each case, one coordinate connects a pair of particles, and the second coordinate connects the third particle to the center of mass of the pair. Capital letters denote heavy-particle coordinates and lower case denote those of the electron. In addition we find it useful to define $\overrightarrow{\mathrm{r}}_{g}$, the electronic vector coordinate measured from the geometric center of the nuclei.

Table I summarizes the relationships between these coordinates, and in Table II we have listed and defined the corresponding reduced masses. We also define the mass asymmetry parameter

$$
\lambda=\left(M_{A}-M_{B}\right) /\left(M_{A}+M_{B}\right), \quad 0 \leqslant \lambda<1 .
$$

Channels are, as usual, the regions of configuration space corresponding to initial and final states of the system, when the $A$ and $B$ subsystems are separated: e.g., the $A$ channel is the region in which the electron is close to nucleus $A$ and both are far from $B$; obviously the system configuration is then properly described using the $\left(\overrightarrow{\mathrm{r}}_{A}, \overrightarrow{\mathrm{R}}_{A}\right)$ coordinates. The molecular region is the part of configuration space in which all three particles are close together and the electron interacts with both centers; in this region the molecular coordinates $(\vec{r}, \vec{R})$ are evidently convenient. In defining these regions we do not wish to imply that any sharp boundary can be drawn which separates them; indeed, the channels can be conceived, at least approximately, as asymptotic portions of the molecular region, and in PSS theory this is how they are treated [using the coordinates $(\vec{r}, \vec{R})$ ]. 
TABLE II. Reduced masses corresponding to coordinates.

\begin{tabular}{lll}
\hline \hline Coordinate & & Reduced mass \\
\hline $\overrightarrow{\mathrm{r}}^{0}$ & $m_{0}$ & Rest mass of electron \\
$\overrightarrow{\mathrm{R}}_{A}^{0}$ & $M_{A}$ & Rest mass of $A$ nucleus \\
$\overrightarrow{\mathrm{R}}_{B}^{0}$ & $M_{B}$ & Rest mass of $B$ nucleus \\
$\overrightarrow{\mathrm{R}}_{\mathrm{c} \cdot \mathrm{m} .}$ & $M_{T}=M_{A}+M_{B}+m_{0}$ & Total mass of system \\
$\overrightarrow{\mathrm{r}}_{A}$ & $m_{A}=m_{0} M_{A} /\left(m_{0}+M_{A}\right)$ & Electron reduced mass, channel $A$ \\
$\overrightarrow{\mathrm{R}}_{A}$ & $\mu_{A}=\left(m_{0}+M_{A}\right) M_{B} / M_{T}$ & Nuclear reduced mass, channel $A$ \\
$\overrightarrow{\mathrm{r}}_{B}$ & $m_{B}=m_{0} M_{B} /\left(m_{0}+M_{B}\right)$ & Electron reduced mass, channel $B$ \\
$\overrightarrow{\mathrm{R}}_{B}$ & $\mu_{B}=\left(m_{0}+M_{B}\right) M_{A} / M_{T}$ & Nuclear reduced mass, channel $B$ \\
$\overrightarrow{\mathrm{r}}^{\overrightarrow{\mathrm{R}}}$ & $m_{0}=m_{0}\left(M_{A}+M_{B}\right) / M_{T}$ & Molecular electron reduced mass \\
$\mu=M_{A} M_{B} /\left(M_{A}+M_{B}\right)$ & Molecular nuclear reduced mass \\
\hline \hline
\end{tabular}

\section{B. Kinematics}

The Schrödinger equation describing relative motion is

$$
H \Psi=(T+V) \Psi=E \Psi .
$$

In the various Jacobi coordinates, $T$ is represented by

$$
\begin{aligned}
& T=-\left(\hbar^{2} / 2 \mu_{A}\right) \vec{\nabla}_{R_{A}}^{2}-\left(\hbar^{2} / 2 m_{A}\right) \vec{\nabla}_{r_{A}}^{2}, \\
& T=-\left(\hbar^{2} / 2 \mu_{B}\right) \vec{\nabla}_{R_{B}}^{2}-\left(\hbar^{2} / 2 m_{B}\right) \vec{\nabla}_{r_{B}}^{2}, \\
& T=-\left(\hbar^{2} / 2 \mu\right) \vec{\nabla}_{R}^{2}-\left(\hbar^{2} / 2 m\right) \vec{\nabla}_{r}^{2} .
\end{aligned}
$$

There are also two unconventional but perfectly valid forms for $T$ which appear later: If we use $\overrightarrow{\mathrm{R}}_{B}$ and $\overrightarrow{\mathrm{r}}$ as the two independent variables, then we obtain from Table I, using the chain rule for partial derivatives,

$$
T=-\frac{\hbar^{2}}{2 \mu_{B}} \vec{\nabla}_{R_{B}}^{2}-\frac{\hbar^{2} m_{0}}{m\left(M_{B}+m_{0}\right)} \vec{\nabla}_{R_{B}} \cdot \vec{\nabla}_{r}-\frac{\hbar^{2}}{2 m} \vec{\nabla}_{r}^{2}
$$

where in this equation $\vec{\nabla}_{r}$ is taken keeping $\overrightarrow{\mathrm{R}}_{B}$ fixed. Similarly, if we use $\overrightarrow{\mathrm{R}}_{A}$ and $\overrightarrow{\mathrm{r}}$ as independent variables, we obtain

$$
T=-\frac{\hbar^{2}}{2 \mu_{A}} \vec{\nabla}_{R_{A}}^{2}+\frac{\hbar^{2} m_{0}}{m\left(M_{A}+m_{0}\right)} \vec{\nabla}_{R_{A}} \cdot \vec{\nabla}_{r}-\frac{\hbar^{2}}{2 m} \vec{\nabla}_{r}^{2} .
$$

\section{Channel eigenstates and scattering boundary conditions}

The wave function $\Psi$ satisfies the usual scatter ing boundary conditions ${ }^{8}$ : in one channel there is a plane wave with the electron in the initial electronic eigenstate, and a set of outgoing spherical waves associated with the electronic states for this channel, while in the other channel there are only outgoing spherically scattered waves associated with various electronic states for that channel.
Channel eigenstates may be defined as follows. The potential $\mathrm{V}$ is assumed to have properties such that in channel $A$,

$$
\lim _{\left(R_{A \rightarrow \infty}, r_{A} \text { fixed }\right)}[V]=V_{0}^{A}\left(\vec{r}_{A}\right)
$$

while in channel $B$,

$$
\lim _{\left(R_{B} \rightarrow \infty, r_{B} f 1 x e d\right)}[V]=V_{0}^{B}\left(\vec{r}_{B}\right) .
$$

We may then define the channel Hamiltonians

$$
\begin{aligned}
& h_{0}^{A}=-\left(\hbar^{2} / 2 m_{A}\right) \vec{\nabla}_{r_{A}}^{2}+V_{0}^{A}\left(\vec{r}_{A}\right), \\
& h_{0}^{B}=-\left(\hbar^{2} / 2 m_{B}\right) \vec{\nabla}_{r_{B}}^{2}+V_{0}^{B}\left(\vec{r}_{B}\right),
\end{aligned}
$$

and their electronic eigenfunctions and eigenvalues

$$
\begin{aligned}
& h_{0}^{A} \phi_{n_{A}}^{0}=\epsilon_{n_{A}}^{0} \phi_{n_{A}}^{0}, \\
& h_{0}^{B} \phi_{n_{B}}^{0}=\epsilon_{n_{B}}^{0} \phi_{n_{B}}^{0} .
\end{aligned}
$$

Channel wave numbers $k_{n_{A}}, k_{n_{B}}$ are given by the relations

$$
\begin{aligned}
& \hbar^{2} k_{n_{A}}^{2} / 2 \mu_{A}+\epsilon_{n_{A}}^{0}=E, \\
& \hbar^{2} k_{n_{B}}^{2} / 2 \mu_{B}+\epsilon_{n_{B}}^{0}=E .
\end{aligned}
$$

Let us further define explicit Cartesian and spherical components for each of the heavy-particle coordinates:

$$
\begin{aligned}
& \overrightarrow{\mathrm{R}}_{A}-\left(X_{A}, Y_{A}, Z_{A}\right) \text { or }\left(R_{A}, \Theta_{A}, \Phi_{A}\right), \\
& \overrightarrow{\mathrm{R}}_{B}-\left(X_{B}, Y_{B}, Z_{B}\right) \text { or }\left(R_{B}, \Theta_{B}, \Phi_{B}\right), \\
& \overrightarrow{\mathrm{R}}-(X, Y, Z) \text { or }(R, \Theta, \Phi) .
\end{aligned}
$$

We can now write down the boundary conditions for a scattering problem. For example, if $A$ is the incident channel, then at large $R_{A}$, finite $r_{A}$,

$$
\begin{aligned}
\Psi \sim & \Phi_{i_{A}}^{0}\left(\overrightarrow{\mathrm{r}}_{A}\right) \exp \left(i k_{i_{A}} Z_{A}\right) \\
& +\sum_{j_{A}} \Phi_{j_{A}}^{0}\left(\mathrm{r}_{A}\right) f_{j_{A} i_{A}}\left(\Theta_{A}, \Phi_{A}\right)\left[R_{A}^{-1} \exp \left(i k_{j_{A}} R_{A}\right)\right],
\end{aligned}
$$


while at large $R_{B}$, finite $r_{B}$,

$$
\Psi \sim \sum_{j_{B}} \Phi_{j_{B}}^{0}\left(\overrightarrow{\mathrm{r}}_{B}\right) f_{j_{B} i_{A}}\left(\Theta_{B}, \Phi_{B}\right)\left[R_{B}^{-1} \exp \left(i k_{j_{B}} R_{B}\right)\right]
$$

It should be noted that even when the heavy particles are electrically identical, i.e., the channel potentials $V_{0}^{A}$ and $V_{0}^{B}$ are identical functions of their respective arguments, the channel eigenstates and eigenvalues will still not be identical if $M_{A} \neq M_{B}$, because $m_{A}$ and $m_{B}$ will differ slightly (cf. Table II). Likewise, the channel wave numbers will differ slightly, not only because $\epsilon_{n_{A}}^{0} \neq \epsilon_{n_{B}}^{0}$ but also (even if we neglected that difference) because $\mu_{A} \neq \mu_{B}$. These small differences are the source of $(g, u)$ couplings in isotopic molecules.

\section{Born-Oppenheimer basis functions}

The Born-Oppenheimer basis functions $\phi_{n}(\overrightarrow{\mathrm{r}} ; \overrightarrow{\mathrm{R}})$ are the eigenfunctions of the molecular electronic Hamiltonian

$$
h_{B O}(\overrightarrow{\mathrm{r}} ; \overrightarrow{\mathrm{R}}) \phi_{n}(\overrightarrow{\mathrm{r}} ; \overrightarrow{\mathrm{R}})=\epsilon_{n}(R) \Phi_{n}(\overrightarrow{\mathrm{r}} ; \overrightarrow{\mathrm{R}})
$$

where

$$
h_{B O}=-\left(\hbar^{2} / 2 m\right) \vec{\nabla}_{r}^{2}+V(\overrightarrow{\mathbf{r}} ; \overrightarrow{\mathbf{R}}) 。
$$

$\left[\Phi_{n}(\vec{r} ; \vec{R})\right.$ is written as function of the vector $\vec{R}$ to indicate explicitly that this function is oriented with respect to the internuclear axis.]

The molecular Hamiltonian $h_{B O}$ differs from the atomic (channel) Hamiltonians $h_{A}^{0}, h_{B}^{0}$ : (i) It contains the full potential $V(\vec{r} ; \vec{R})$, not just an asymptotic projection of it; (ii) the molecular electronic reduced mass, $m$, appears in the kinetic energy term. At larger $R$, however, there is an approximate equality between the Born-Oppenheimer eigenstates and the atomic eigenstates.

We say a system is symmetric if the two atomic potentials $V_{0}^{A}, V_{0}^{B}$ are identical functions of their respective arguments. In this case the BornOppenheimer eigenfunctions are also parity eigenfunctions $(g, u)$. If in addition $M_{A}=M_{B}$, we say the system is homonuclear (otherwise it is heteronuclear).

In the asymmetric case, each Born-Oppenheimer function is uniquely correlated to an atomic eigenfunction in one channel or the other (assuming no accidental asymptotic degeneracy); either

$\lim _{\left(R \rightarrow \infty, r_{A} \text { fixed }\right)}\left[\phi_{n}(\overrightarrow{\mathrm{r}} ; \overrightarrow{\mathrm{R}})\right]=\phi_{n_{A}}\left(\overrightarrow{\mathrm{r}}_{A}\right) \simeq \phi_{n_{A}}^{0}\left(\overrightarrow{\mathrm{r}}_{A}\right)$

and

$\lim _{\left(R \rightarrow \infty, r_{B} \text { fixed }\right)}\left[\phi_{n}(\overrightarrow{\mathrm{r}} ; \overrightarrow{\mathrm{R}})\right]=0$,

with $\lim _{R \rightarrow \infty}\left[\epsilon_{n}(R)\right]=\epsilon_{n_{A}} \simeq \epsilon_{n_{A}}^{0}$

or else

$\lim _{\left(R \rightarrow \infty, r_{A} \mathrm{fixed}\right)}\left[\phi_{n}(\overrightarrow{\mathrm{r}} ; \overrightarrow{\mathrm{R}})\right]=0$

and

$\lim _{\left(R \rightarrow \infty, r_{B} \text { fixed }\right)}\left[\phi_{n}(\overrightarrow{\mathrm{r}} ; \overrightarrow{\mathrm{R}})\right]=\phi_{n_{B}}\left(\overrightarrow{\mathrm{r}}_{B}\right) \simeq \phi_{n_{B}}^{0}\left(\overrightarrow{\mathrm{r}}_{B}\right)$,

with

$\lim _{R \rightarrow \infty}\left[\epsilon_{n}(R)\right]=\epsilon_{n_{B}} \simeq \epsilon_{n_{B}}^{0}$.

These correspondences are approximate rather than exact because the molecular electron mass $m$ differs from the atomic ones $m_{A}, m_{B}$ (cf. Table II).

For the symmetric case (whether or not it is homonuclear) the Born-Oppenheimer eigenfunctions correlate to $g$ and $\underline{u}$ linear combinations of (approximate) atomic orbitals. For $\underline{g}$ states,

$$
\begin{aligned}
\quad \lim _{\left(R \rightarrow \infty, r_{A} \text { fixed }\right)}\left[\phi_{n}(\overrightarrow{\mathrm{r}} ; \overrightarrow{\mathrm{R}})\right] \\
=(1 / \sqrt{2}) \phi_{n_{A}}\left(\overrightarrow{\mathrm{r}}_{A}\right) \simeq(1 / \sqrt{2}) \phi_{n_{A}}^{0}\left(\overrightarrow{\mathrm{r}}_{A}\right)
\end{aligned}
$$

and

$$
\begin{aligned}
& \lim _{\left(R \rightarrow \infty, r_{B} \text { f1xed }\right)}\left[\phi_{n}(\overrightarrow{\mathrm{r}} ; \overrightarrow{\mathrm{R}})\right] \\
& =(1 / \sqrt{2}) \phi_{n_{B}}\left(\overrightarrow{\mathrm{r}}_{B}\right) \simeq(1 / \sqrt{2}) \phi_{n_{B}}^{0}\left(\overrightarrow{\mathrm{r}}_{B}\right) .
\end{aligned}
$$

For $u$ states,

$$
\begin{aligned}
\lim _{\left(R \rightarrow \infty, r_{A} \text { fixed }\right)}\left[\phi_{n}(\overrightarrow{\mathrm{r}} ; \overrightarrow{\mathrm{R}})\right] \\
\quad=-(1 / \sqrt{2}) \phi_{n_{A}}\left(\overrightarrow{\mathrm{r}}_{A}\right) \simeq-(1 / \sqrt{2}) \phi_{n_{A}}^{0}\left(\overrightarrow{\mathrm{r}}_{A}\right),
\end{aligned}
$$

and

$$
\begin{aligned}
& \lim _{\left(R \rightarrow \infty, r_{B} \text { fixed }\right)}\left[\phi_{n}(\overrightarrow{\mathrm{r}} ; \overrightarrow{\mathrm{R}})\right] \\
& \quad=(1 / \sqrt{2}) \phi_{n_{B}}\left(\overrightarrow{\mathrm{r}}_{B}\right) \simeq(1 / \sqrt{2}) \phi_{n_{B}}^{0}\left(\overrightarrow{\mathrm{r}}_{B}\right) .
\end{aligned}
$$

For both parities, $n_{A}$ and $n_{B}$ denote the same index, and

$$
\lim _{R \rightarrow \infty}\left[\epsilon_{n}(R)\right] \equiv \epsilon_{n} \simeq \epsilon_{n_{A}}^{0} \simeq \epsilon_{n_{B}}^{0} .
$$

\section{REACTION COORDINATES}

\section{A. Mass-scaled coordinates}

Mass-scaled coordinates have been used for many years to study reactive collisions,, 90 especially for collinear atom-diatom collisions. We define our mass-scaled coordinates by

$$
\text { (associated mass })^{1 / 2} \times^{*}(\text { coordinate }),
$$

where the associated masses are given in Table II; thus, denoting the mass-scaled coordinates by a tilde, 

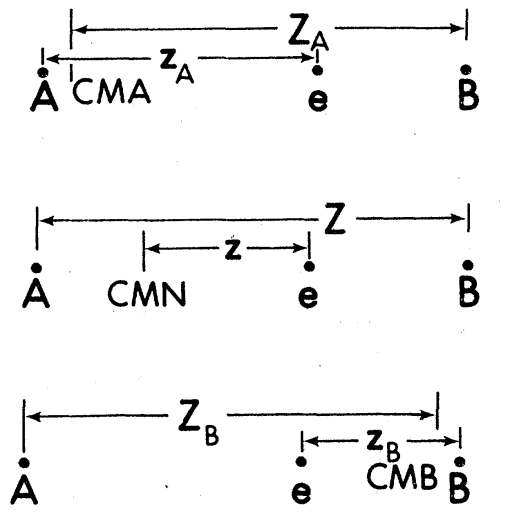

FIG. 2. Coordinates for collinear collisional model.

$$
\overrightarrow{\overrightarrow{\mathrm{r}}}_{A}=\left(m_{A}\right)^{1 / 2} \overrightarrow{\mathrm{r}}_{A}, \quad \overrightarrow{\tilde{\mathrm{R}}}_{A}=\left(\mu_{A}\right)^{1 / 2} \overrightarrow{\mathrm{R}}_{A},
$$

and so on. The conjugate momenta are transformed contragradiently, i.e., if $\vec{p}_{A}$ is conjugate to $\overrightarrow{\mathrm{r}}_{A}$, then $\overrightarrow{\tilde{\mathrm{p}}}_{A}=\left(m_{A}\right)^{-1 / 2} \overrightarrow{\mathrm{p}}_{A}$, and so on; the transformation between scaled and unscaled coordinates is then canonical. The transformed kinetic energy operators from Eqs. (2.3) are given by

$$
\begin{aligned}
T=-\frac{1}{2} \hbar^{2}\left[\vec{\nabla}_{\tilde{R}_{A}}^{2}+\vec{\nabla}_{\tilde{r}_{A}}^{2}\right] & =-\frac{1}{2} \hbar^{2}\left[\vec{\nabla}_{\tilde{R}_{B}}^{2}+\vec{\nabla}_{\tilde{r}_{B}}^{2}\right] \\
& =-\frac{1}{2} \hbar^{2}\left[\vec{\nabla}_{\tilde{R}}^{2}+\vec{\nabla}_{\tilde{r}}^{2}\right],
\end{aligned}
$$

so that we may now think of the collision as the motion of a single particle (of unit mass) on a six-dimensional potential surface.

We can form a useful intuitive picture of our

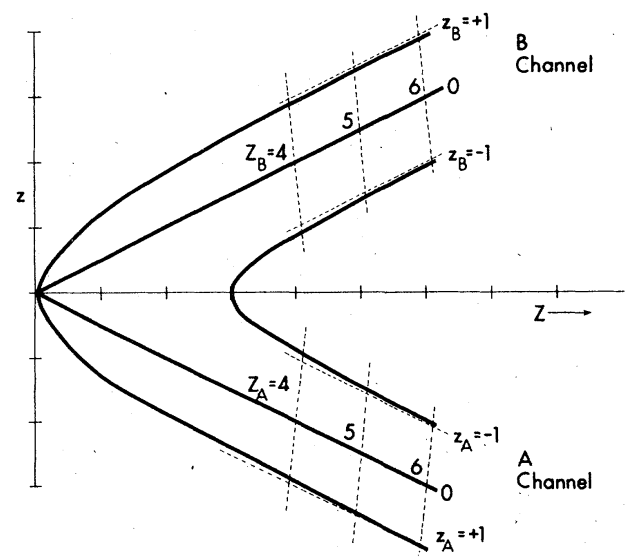

FIG. 3. Atomic and molecular coordinates and equipotential lines for collision on the collinear cut, for hydrogenic nuclei of equal mass. Heavy solid lines denote the equipotentials $V=-1,-\infty,-1$, marking centers and approximate width of the channels. The dotted lines mark lines of constant $Z_{A}, z_{A}$ or $Z_{B}, z_{B}$. Masses $M_{A}=M_{B}=9, m_{0}=1$ for this picture. problem with a two-dimensional model. Consider the collinear cut of the potential surface, and think about the motion in the $(\tilde{z}, \tilde{Z})$ plane (Fig. 2). We imagine that the electron may pass the nuclei, but the nuclei may not pass each other; hence $\tilde{Z} \geqslant 0$, but $\tilde{z}_{A}, \tilde{z}_{B}$, and $\tilde{z}$ may be either positive or negative. In Fig. 3, we have depicted the equipotential lines (for this two-dimensional collinear cut of the potential surface), as a function of the unscaled variables $(z, Z)$; the system is homonuclear and the potential due to each nucleus is Coulombic with unit nuclear charge. At $z= \pm \frac{1}{2} Z$ are attractive singularities defining the centers of the channels, and the channels may be considered to be the asymptotic regions at larger $Z$ which are bounded by $\left|z \mp \frac{1}{2} Z\right| \simeq$ (a few a.u.)

In Fig. 4 the same picture is shown in massscaled coordinates $(\tilde{z}, \tilde{Z})$ for a hypothetical "molecule" with $M_{A}=M_{B}=9, m_{0}=1$. The angle between the channels is now smaller [for real molecules, it will be very tiny indeed, of order $\left.(m / \mu)^{1 / 2}\right]$. Since the figure is drawn with $\tilde{z}$ and $\tilde{Z}$ orthogonal, we now find that $\left(\tilde{z}_{A}, \tilde{Z}_{A}\right)$ and $\left(\tilde{z}_{B}, \tilde{Z}_{B}\right)$ also form orthogonal coordinates, and we can see pictorially the well-known result that the atomic and molecular mass-scaled coordinates are related by simple rotations.

The situation for a symmetric system with nonidentical nuclei is depicted in Figs. 5 and 6 . The potentials are again the Coulombic ones of the previous case, but the masses now are $M_{A}=18$, $M_{B}=6, m_{0}=1$. Even in unscaled coordinates $(z, Z)$ (Fig. 5), the picture is skewed off-axis because $z$ is measured not from the geometric center of the nuclei, but from the center of mass of the nuclei (CMN); the angle between the channels is bisected not by the $Z$ axis $(z=0)$, but by the line $z_{g}=z-\frac{1}{2} \lambda Z$ $=0$. In mass-scaled coordinates $(\tilde{z}, \tilde{Z})$ (Fig. 6), the

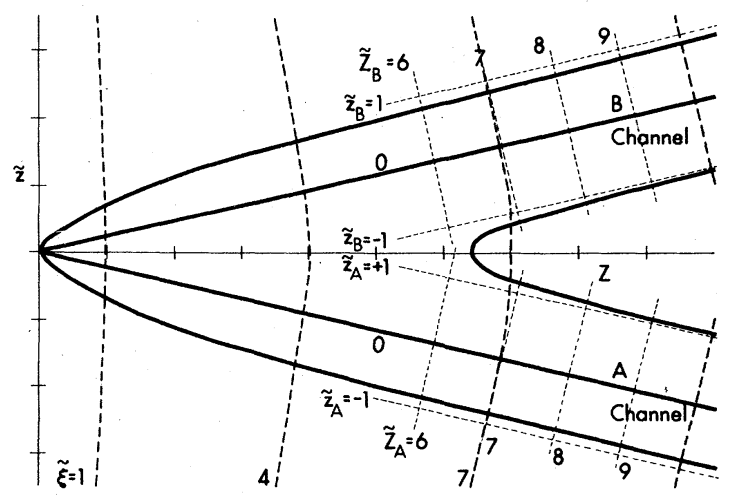

FIG. 4. Corresponding figure to Fig. 3, drawn with mass-scaled coordinates $z, \tilde{Z}$. Dashed curves are lines of constant $\overrightarrow{\tilde{\xi}}$ (cf. Sec. III C). 


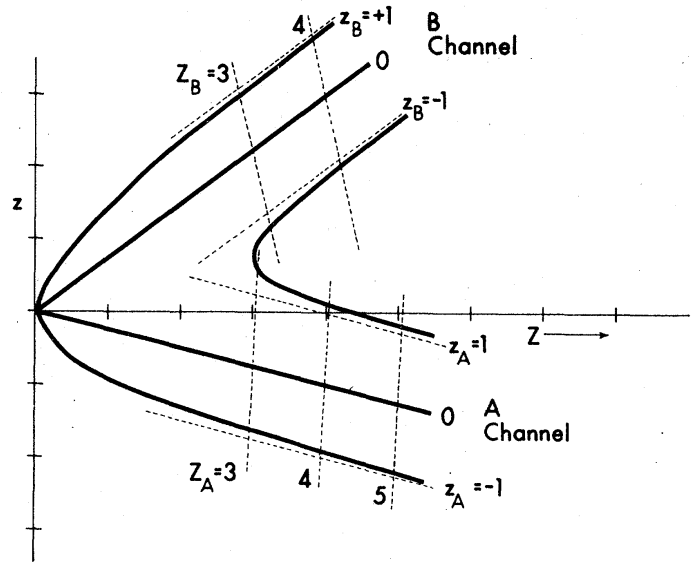

FIG. 5. Same as Fig. 3, but for nuclei of unequal mass $M_{A}=18, M_{B}=6$.

bisector of the channels is still off-axis, but once again the atomic and molecular mass-scaled coordinates all form orthogonal coordinate pairs and are related to each other by simple rotations.

Analogous pictures would be obtained from other cuts of the potential surface, and, though these figures are necessarily restricted to two dimensions, they provide insights applicable to the full six-dimensional situation. We shall refer to these pictures to clarify our discussion at later points. (All equations written in the text, of course, refer to the full six-dimensional problem.)

The rotations which relate the mass-scaled molecular coordinates to the mass-scaled atomic channel coordinates can be obtained by straightforward calculation from Tables I and II and Eq. (3.1):

$$
\begin{aligned}
& {\left[\begin{array}{l}
\overrightarrow{\tilde{\mathrm{r}}}_{A} \\
\overrightarrow{\tilde{\mathrm{R}}}_{A}
\end{array}\right]=\left[\begin{array}{cc}
\cos \sigma_{A} & -\sin \sigma_{A} \\
\sin \sigma_{A} & \cos \sigma_{A}
\end{array}\right]\left[\begin{array}{c}
\overrightarrow{\tilde{\mathrm{r}}} \\
\overrightarrow{\overrightarrow{\mathrm{R}}}
\end{array}\right],} \\
& {\left[\begin{array}{l}
\overrightarrow{\tilde{\mathrm{r}}}_{B} \\
\overrightarrow{\tilde{\mathrm{R}}}_{B}
\end{array}\right]=\left[\begin{array}{ll}
\cos \sigma_{B} & -\sin \sigma_{B} \\
\sin \sigma_{B} & \cos \sigma_{B}
\end{array}\right]\left[\begin{array}{c}
\overrightarrow{\tilde{\mathrm{r}}} \\
\overrightarrow{\tilde{\mathrm{R}}}
\end{array}\right],}
\end{aligned}
$$

where

$$
\begin{aligned}
\tan \sigma_{A} & =-\frac{1}{2}(1-\lambda)(m / \mu)^{1 / 2} \\
& =-\left(m_{0} / M_{T}\right)^{1 / 2}\left(M_{B} / M_{A}\right)^{1 / 2} \\
\cos \sigma_{A} & =\left(m_{A} / m\right)^{1 / 2} \\
& =\left(\mu_{A} / \mu\right)^{1 / 2}\left[\left(M_{A}+\frac{1}{2}(1+\lambda) m_{0}\right) /\left(M_{A}+m_{0}\right)\right]
\end{aligned}
$$

$$
\begin{aligned}
\sin \sigma_{A} & =-\frac{1}{2}(1-\lambda)\left(m_{A} / \mu\right)^{1 / 2} \\
& =-\left(\mu_{A} / m\right)^{1 / 2}\left[m_{0} /\left(M_{A}+m_{0}\right)\right],
\end{aligned}
$$

and

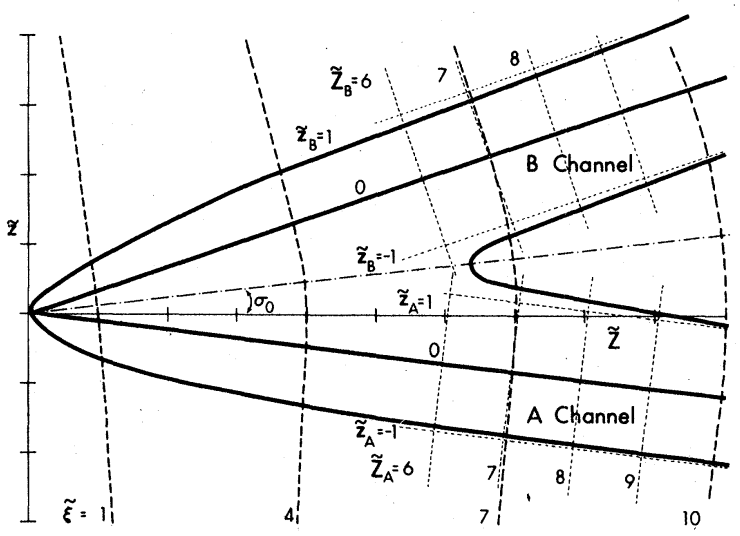

FIG. 6. Analog to Fig. 4 for the case of unequal masses. The line bisecting the angle between channels is the geometric center of the nuclei; it is rotated by an angle $\sigma_{0}$ with respect to the $Z$ axis [cf. Eq. (3.13c)]

$$
\begin{aligned}
\tan \sigma_{B} & =\frac{1}{2}(1+\lambda)(m / \mu)^{1 / 2} \\
& =\left(m_{0} / M_{T}\right)^{1 / 2}\left(M_{A} / M_{B}\right)^{1 / 2}, \\
\cos \sigma_{B} & =\left(m_{B} / m\right)^{1 / 2} \\
& =\left(\mu_{B} / \mu\right)^{1 / 2}\left\{\left[M_{B}+\frac{1}{2}(1-\lambda) m_{0}\right] /\left(M_{B}+m_{0}\right)\right\},
\end{aligned}
$$

$$
\begin{aligned}
\sin \sigma_{B} & =\frac{1}{2}(1+\lambda)\left(m_{B} / \mu\right)^{1 / 2} \\
& =\left(\mu_{B} / m\right)^{1 / 2}\left[m_{0} /\left(M_{B}+m_{0}\right)\right] .
\end{aligned}
$$

\section{B. Properties of scattering coordinates}

We have described the three coordinate systems which are appropriate, respectively, to the $A$ and $B$ atomic channels and to the molecular region; we have shown how they are related to each other (when expressed in mass-scaled form) by simple rotations, and we have used the two-dimensional cuts shown in Figs. 3-6 to create an intuitive picture of the relation between them. Now our purpose is to define a new pair of coordinates $(\vec{\xi}, \vec{\eta})$ which is locally adapted to each of the channels and to the molecular region as well; $\vec{\xi}$ will be the heavy-particle coordinate, or scattering coordinate, while $\vec{\eta}$ is the electron coordinate. How ever, the definition of "reaction coordinates" which is appropriate to this problem is quite different, both in its form and its physical premises, from those familiar in the theory of nuclear reactions and chemical rearrangments. Our own approach can be made clearer if we examine some of the ideas involved in these more familiar theories by way of contrast. Each approach appeals to pictures similar to Figs. 3-6, but draws different conclusions from them. 


\section{1. "Collision complex" methods}

Many types of nuclear collisions and some chemical reactions can be studied conveniently by drawing sharp boundaries between the "channels" and the "collision complex," solving the Schrödinger equation using the coordinate system and the solution method most suitable for each region, and joining the solutions at the boundaries. This is the approach of the $R$-matrix method in nuclear collision theory ${ }^{11}$ and it is similar in spirit to some numerical methods being used to study chemical reactivity. ${ }^{12}$ While these methods can be made formally exact, they seem to be most useful if (a) there is a well-defined boundary between "channels" and "complex" (or "reactants" and "products"), and/or (b) the "collision complex" Hamiltonian and eigenstates are quite unlike those of the "channels." Neither of these features marks slow atomic collisions. Electronic states alter continuously from "molecular" to "atomic" character as the internuclear separation increases; even in the heart of the molecular region, the local electronic wave function near a nucleus is not unlike an atomic state [as the linear combination of atomic orbitals (LCAO) approximation assumes], while conversely the atomic states are quite closely approximated for most purposes by the molecular electronic eigenstates, a fact which is the basis of PSS theory. Therefore, instead of using discontinuously different. regions, we seek a single, continuously changing coordinate system, which asymptotically matches the channel coordinates in each channel, yet is appropriate in the molecular region. The molecular coordinates $(\vec{R}, \overrightarrow{\mathbf{r}})$ themselves would be suitable for this purpose if the PSS theory were an adequate one, but as it is just the defects of PSS theory that we wish to remedy, we must do better than the choice $(\vec{R}, \overrightarrow{\mathrm{r}})$, not only in the asymptotic channels but in the molecular region.

\section{2. "Reaction path" methods of chemical dynamics}

Underlying the use of reaction coordinates in chemical dynamics ${ }^{13}$ - as, for example, in the paradigm reaction of an atom with a diatom - is an attempt to identify "translational" and "internal" degrees of freedom, in such a way that the translational coordinate is a progress variable along the path from reactants to products. In this approach, the curve associated with the reaction path comes in (say) along the $A$ channel, "turns the corner," and goes out the $B$ channel; the value of the coordinate on the path tells "how far" the system has proceeded from one channel to the other. The remaining "internal" coordinate is defined in a manner locally specified by the value of the path variable; it, too, twists around the corner, following the reaction path curve and being orthogonal to it at each point.

Given the formal equations (3.3), the procedure for constructing this type of reaction coordinate transformation is clear enough. All we would have to do is define a progress variable, $\zeta$, say, which runs from $-\infty$ in asymptotic channel $A$, through zero in the collision region, out to $+\infty$ in asymptotic channel $B$; then we would define a single-valued, continuously varying angle $\sigma(\zeta)$, $\sigma_{A} \leqslant \sigma(\zeta) \leqslant \sigma_{B}$, which specifies a transformation of the sameform as Eqs. (3.3). In channel $A$, the asymptotic reaction path coordinate is clearly related to $\overrightarrow{\mathrm{R}}_{A}$, while in channel $B$ it becomes $\overrightarrow{\mathrm{R}}_{B}$, and so on. However, this type of transformation is entirely inappropriate to our problem.

Such a transformation does not provide a oneto-one mapping. A given point on the potential surface (most obviously, on the ridge separating the two channels) corresponds to two or more distinct values of the path variable and its locally defined orthogonal "internal" coordinate. In chemical dynamics, this defect presents no serious difficulty because one supposes that the channel "valleys" are very deep, and hence internal motion transverse to the reaction path is locally confined to that particular valley; regions up on the ridge where the definition of coordinates is seriously ambiguous are classically forbidden and effectively play no part in the dynamics. Such an idea, that the value of the reaction path variable fully specifies a local coordinate system, is only sensible if it is indeed the case that motion in the other degrees of freedom really is locally confined. But in a slow atomic collision this is not the case. The electron is delocalized over the entire system, tunneling rapidly through the ridge between channels; in no way can its configuration be confined in one channel or the other as a specified function of some progressive path variable.

The scattering coordinate transformation we shall devise here embodies an idea which is almost the converse of the reaction path concept. Instead of defining an electron coordinate locally specified by a reaction coordinate of some kind, we will instead define a scattering coordinate $\vec{\xi}$ which is functionally dependent upon the electron's position. The transformation will be guaranteed to be a one-to-one mapping by ensuring that the electron coordinate is uniquely defined, while the scattering coordinate $\vec{\xi}$ is a single-valued function of $(\vec{R}, \vec{r})$. We begin, however, with the definition of $\vec{\xi}$, which plays the crucial role in the theory, and subsequently consider the definition of the electron coordinate $\vec{\eta}$. 


\section{Definition of heavy-particle coordinate $\vec{\xi}$}

To define a (mass-scaled) scattering coordinate which depends upon the electron position, we begin with the formal relation

$$
\overrightarrow{\tilde{\xi}}=\overrightarrow{\tilde{R}} \cos \sigma(\overrightarrow{\mathrm{r}} ; \overrightarrow{\mathrm{R}})+\overrightarrow{\overrightarrow{\mathrm{r}}} \sin \sigma(\overrightarrow{\mathrm{r}} ; \overrightarrow{\mathrm{R}}),
$$

a choice obviously inspired by the second rows of Eqs. (3.3a) and (3.3b); if we then arrange things so that the angle $\sigma(\overrightarrow{\mathrm{r}} ; \overrightarrow{\mathrm{R}})$ approaches $\sigma_{A}$ when the electron is near nucleus $A$, and approaches $\sigma_{B}$ when it is near nucleus $B$, then correspondingly $\vec{\xi}$ will approach $\overrightarrow{\mathrm{R}}_{A}$ or $\overrightarrow{\mathrm{R}}_{B}$. The required proper ties are obtained by again employing the switching function $f(\overrightarrow{\mathrm{r}} ; \overrightarrow{\mathrm{R}})$, which was previously introduced in ETF theory in a quite different formal context [Eqs. (2.34) of Ref. 1]. It has the asymptotic properties

$$
\lim _{\left(R \rightarrow \infty, r_{A}\right. \text { finite) }}[f(\overrightarrow{\mathrm{r}} ; \overrightarrow{\mathrm{R}})]=-1
$$

and

$$
\lim _{\left(R \rightarrow \infty, r_{B} \text { finite }\right)}[f(\overrightarrow{\mathrm{r}} ; \overrightarrow{\mathrm{R}})]=+1,
$$

but is not yet further defined. However, our experience from ETF theory suggests that even for finite $R$ values (i.e., in the molecular region) $f$ will assume values near -1 in the vicinity of nucleus $A$, and values near +1 in the vicinity of nucleus $B$. Then we write

$$
\tan \sigma(\overrightarrow{\mathrm{r}} ; \overrightarrow{\mathrm{R}})=\frac{1}{2}[f(\overrightarrow{\mathrm{r}} ; \overrightarrow{\mathrm{R}})+\lambda](m / \mu)^{1 / 2},
$$

and $\sigma(\vec{r} ; \vec{R})$ is seen to have the desired properties. $\vec{\xi}$ goes to $\overrightarrow{\mathrm{R}}_{A}$ in the asymptotic $A$ channel, and to $\overrightarrow{\mathrm{R}}_{B}$ in the asymptotic $B$ channel; in the molecular region it can also approach these quantities when the electron is near the respective nuclei.

To illustrate this definition, we have depicted lines of constant $\tilde{\xi}$ in the two dimensional cuts of Figs. 4 and 6; these have been calculated using the switching function determined by Lebeda, Thorson and Levy ${ }^{14}$ for the $1 s \sigma_{g}$ state of $\mathrm{H}_{2}^{+}$(via the ETF formulation of the theory).

In the rest of this paper we will systematically develop coupled equations which include all effects accurate to terms of order $(m / \mu)$. For this pur pose we may expand Eq. (3.6) for small $\sigma$, and for the unscaled scattering coordinate we obtain

$$
\vec{\xi} \equiv \overrightarrow{\tilde{\xi}} / \mu^{1 / 2}=\overrightarrow{\mathbf{R}}+(m / \mu) \overrightarrow{\mathbf{s}}(\overrightarrow{\mathbf{r}} ; \overrightarrow{\mathbf{R}})+O(m / \mu)^{2},
$$

with

$$
\overrightarrow{\mathrm{s}}(\overrightarrow{\mathrm{r}} ; \overrightarrow{\mathrm{R}})=\frac{1}{2}[f(\overrightarrow{\mathrm{r}} ; R)+\lambda] \overrightarrow{\mathrm{r}}-[f(\overrightarrow{\mathrm{r}} ; R)+\lambda]^{2} \overrightarrow{\mathrm{R}} / 8
$$

or, alternatively,

$$
\overrightarrow{\mathbf{s}}(\overrightarrow{\mathbf{r}} ; \overrightarrow{\mathbf{R}})=\frac{1}{2}[f(\overrightarrow{\mathbf{r}} ; R)+\lambda] \overrightarrow{\mathbf{r}}_{\boldsymbol{g}}-\left(1-\lambda^{2}\right) \overrightarrow{\mathrm{R}} / 8 .
$$

(3.9c) differs from (3.9b) only in the replacement of $f^{2}$ by 1 .

The definition (3.6) [and its approximate form (3.9)] turns out to be the critical step in the theory. Once we decide to use $\vec{\xi}$ as one of the independent variables, and employ a description of the electronic motion in a set of eigenfunctions defined on a surface of constant $\vec{\xi}$, then the rest of the theory, including the correct coupled equations, follows quite directly; the specific definition of the new electronic coordinate $\vec{\eta}$ is less critical (as long as it provides a one-to-one mapping). But we have seen here that once we hit upon the essential idea that $\vec{\xi}$ must depend functionally upon the electron's position, the correct definition for $\overrightarrow{\tilde{\xi}}$ emerges quite naturally from the properties of the massscaled coordinates and the rotations which connect them.

\section{Definition of electron coordinate $\vec{\eta}$}

We now require a second coordinate complementary to $\vec{\xi}$, to specify the system configuration, and since Eq. (3.9a) tells us that $\vec{\xi}$ is essentially a heavy-particle coordinate, $\vec{\eta}$ must be an electronic coordinate.

The most obvious definition at hand is that cor responding to Eq. (3.6) for $\vec{\xi}$, i.e.,

$$
\overrightarrow{\tilde{\eta}}=-\overrightarrow{\tilde{R}} \sin \sigma(\overrightarrow{\mathrm{r}} ; \overrightarrow{\mathrm{R}})+\overrightarrow{\overrightarrow{\mathrm{r}}} \cos \sigma(\overrightarrow{\mathrm{r}} ; \overrightarrow{\mathrm{R}}) \text {. }
$$

This seems very attractive because (a) in the asymptotic channel limits $\overrightarrow{\tilde{\eta}}$ approaches the appropriate channel electronic coordinates $\overrightarrow{\vec{r}}_{A}$ or $\overrightarrow{\tilde{r}}_{B}$, and (b) it obviously forms a locally orthogonal curvilinear system with $\vec{\xi}$. However, it is not an acceptable form because it does not yield a oneto-one mapping; at large $R$, three quite different electron configurations correspond to the same value of $\vec{\eta}$, and attempts to discriminate the three sheets lead right back to a reaction path variable concept which we have already rejected as physically inappropriate.

The definition we shall take is simply

$$
\vec{\eta}=\overrightarrow{\mathbf{r}} \text {. }
$$

From Eq. (3.6), we see that since $\sin \sigma \simeq \sigma$ is of or$\operatorname{der}(m / \mu)^{1 / 2}$ in any case, $\overrightarrow{\tilde{\eta}}$ and $\overrightarrow{\tilde{\xi}}$ are almost orthogonal. Furthermore, since $\vec{\xi}$ becomes the correct heavy-particle coordinate $\overrightarrow{\widetilde{R}}_{A}$ or $\overrightarrow{\tilde{R}}_{B}$ in the asymptotic channels, we then obtain (channel $J=A$ or $B$ )

$$
\overrightarrow{\tilde{\eta}}=\overrightarrow{\tilde{r}}_{J} \cos \sigma_{J}+\vec{\xi} \sin \sigma_{J} ;
$$

for a fixed but asymptotic value of $\vec{\xi}$, $\vec{\eta}$ is thus related to the correct atomic electronic coordinate by a linear scaling. It follows that using $(\vec{\eta}, \vec{\xi})$ as new dynamical variables, we can define a molec- 
ular electronic Hamiltonian which will yield the exact atomic electronic eigenstates for each channel, in the limit of fixed but asymptotically large $|\vec{\xi}|$. We shall define such a Hamiltonian in Sec. IV.

[Actually, provided the transformation $(\vec{r}, \vec{R})-(\vec{\eta}, \vec{\xi})$ is one-to-one, the specific form chosen for $\vec{\eta}$ is not very critical. For example, we could choose either

$$
\text { or } \begin{gathered}
\overrightarrow{\tilde{\eta}}^{\prime}=\overrightarrow{\vec{r}}_{g} \\
\overrightarrow{\vec{\eta}}^{\prime \prime}=-\overrightarrow{\tilde{R}} \sin \sigma_{0}+\overrightarrow{\vec{r}} \cos \sigma_{0},
\end{gathered}
$$

where

$$
\tan \sigma_{0}=\frac{1}{2} \lambda(m / \mu) \text {. }
$$

For these choices, lines of constant $\overrightarrow{\tilde{\eta}}$ lie parallel to the line $\tilde{z}_{g}=0$ in the two-dimensional cut of Fig. 6 , with the choice $(3.13 \mathrm{~b})$ giving a better scaling. However, these choices lead to additional, unnecessary complications in the electronic Hamiltonian, which can be avoided with the form (3.2)].

\section{E. Scheme of derivation}

Development of the theory now proceeds essentially as follows: (i) The Schrödinger equation and the scattering boundary conditions are expressed in terms of the new dynamical variables $(\vec{\eta}, \vec{\xi})$. The boundary conditions are easily expressed since $\vec{\xi}$ takes the correct form in each channel.

(ii) A suitable molecular electronic Hamiltonian and its corresponding complete set of electronic eigenstates and eigenvalues are defined (as functions of the electronic coordinate $\vec{\eta}$ ), on surfaces of fixed $\vec{\xi}$. Such a set can be defined for each value of $\vec{\xi}$, which plays the role merely of a parameter specifying the set.

(iii) The solution to the Schrödinger equation is written as an expansion in the basis set of electronic eigenfunctions. The expansion coefficients are functions of the scattering coordinate $\vec{\xi}$; they meet the scattering boundary conditions and obey a system of coupled differential equations whose proper derivation is the main objective of this paper.

In Sec. IV, we describe two different choices for the molecular electronic Hamiltonian and its eigenstates. One of these is formally important, while the other is more practical. In both cases, we stress the point that these eigenfunctions are defined on a surface of constant $\vec{\xi}$.

In Sec. V, the full Hamiltonian is expressed in the new dynamical variables $(\vec{\eta}, \vec{\xi})$, the scattering wave function is written as an expansion in molecular electronic basis functions, and coupled equa- tions for the heavy-particle wave functions $\chi_{n}(\vec{\xi})$ are derived (for each of the electronic basis sets defined in Sec. IV).

The important difference between this theory and the PSS theory is the introduction of the scattering coordinate $\vec{\xi}$ in place of $\vec{R}$, and the corresponding redefinition of molecular electronic states (of whatever kind) on surfaces of fixed $\vec{\xi}$, rather than fixed $\vec{R}$. The variable transformation then produces some new coupling terms in the Hamiltonian, and there is an important new physical feature: a momentum "in the $\vec{\xi}$ direction" (i.e., perpendicular to surfaces of constant $\vec{\xi}$ ) implicitly includes both the momentum of the heavy particles and the translational momentum of an electron bound to them (this is evident in Figs. 4 and 6). When the full wave function is expanded in an electronic basis set defined on surfaces of fixed $\vec{\xi}$, the "heavy-particle wave functions" $\chi_{n}(\vec{\xi})$ then include the electron translation factor and it does not have to be put in separately as in the preceding paper. ${ }^{1}$ In the formulation of Ref. 1 , the correction matrices $\overrightarrow{\mathbb{A}}$ which modify the PSS matrices $\underline{\vec{P}}$ arise from the ETF's; here they appear instead as a result of the new couplings produced by the coordinate transformation.

Throughout the rest of the paper we pay particular attention at critical points to the test problem of "isotopic molecules."

In what follows, we work wherever possible with the unscaled new variables $\vec{\xi}$ and $\vec{\eta}=\vec{r}$. Exact results will be retained where formally important, but equations for practical use are expanded in powers of the mass ratio $m / \mu$, keeping all terms to order $m / \mu$.

\section{MOLECULAR ELECTRONIC BASIS FUNCTIONS}

We have not yet expressed the complete Hamiltonian in the new variables $(\vec{\xi}, \vec{\eta}=\overrightarrow{\mathrm{r}}$ ) (see Sec. V); however, we expect that, just as in PSS theory, it can be divided into an "electronic part" and a "heavy-particle part." The eigenfunctions of the electronic part will be used as a basis set for expanding the full wave function.

To describe a slow collision properly, such basis functions should be generally adiabatic and molecular in character. However, the essential new property we require of them is that they are defined on a surface of constant $\vec{\xi}$, and that on that surface they form a complete orthogonal set for the expansion of functions in $\overrightarrow{\mathrm{r}}$ space.

In this section, we describe two possible choices for the electronic Hamiltonian and its corresponding complete set of eigenstates on a surface of fixed $\vec{\xi}$. The first of these is elegant and formally important, because its eigenstates $\psi_{n}(\overrightarrow{\mathrm{r}} ; \vec{\xi})$ become 
the exact atomic electronic eigenstates in each asymptotic channel. When we use this set as an expansion basis all formal requirements and boundary conditions are satisfied exactly; this proves that with the present theory we can give an exact formulation of slow atomic collision theory, in which no infinite range couplings or other formal defects of any kind appear. However, this basis set is unfamiliar, difficult to calculate except as an expansion in a more familiar basis, and is of little practical or even physical importance for solving collision problems.

The second electronic Hamiltonian has a set of eigenfunctions which are approximate rather than exact channel eigenstates [approximate in the same way as the Born-Oppenheimer states, Eqs. (2.13)(2.17)], but they are familiar functions and more easily computed. The coupled equations derived in this basis are appropriate for the practical solution of slow collision problems, and they are fully quantum-mechanical equations equivalent to those derived via the ETF formulation [Eqs. (3.7) of Ref. 1].

\section{A. Formally useful electronic basis set}

Let us define a set of electronic states $\psi_{n}(\vec{r} ; \vec{\xi})$ by the equation

$$
h(\overrightarrow{\mathrm{r}} ; \vec{\xi}) \psi_{n}(\overrightarrow{\mathrm{r}} ; \vec{\xi})=W_{n}(\vec{\xi}) \psi_{n}(\overrightarrow{\mathrm{r}} ; \vec{\xi})
$$

with

$$
h=-\left(\hbar^{2} / 2 m\right)(\cos \sigma)\left(\vec{\nabla}_{r}^{2}\right)(\operatorname{\xi }(\cos \sigma)+V,
$$

where $\left(\vec{\nabla}_{r}^{2}\right)_{\xi}$ means the derivative with $\vec{\xi}$ fixed. For reasons that will be clear later, we need to be very careful about functional notation at this point. We write

$$
V=V_{\xi}(\overrightarrow{\mathbf{r}} ; \vec{\xi})=V(\overrightarrow{\mathbf{r}} ; \overrightarrow{\mathbf{R}})
$$

and

$$
\sigma=\sigma_{\xi}(\vec{r} ; \vec{\xi})=\sigma(\vec{r} ; \vec{R})
$$

to represent the potential $V$ and the rotation angle $\sigma$. By this we mean that $V_{\xi}(\vec{r} ; \vec{\xi})$ and $V(\vec{r} ; \vec{R})$ are different functions of their respective arguments, but represent the same physical potential at a given point, the point described as $(\vec{r}, \vec{\xi})$ and as $(\vec{r}, \vec{R})$ in the new and old coordinates respectively [the transformation which links $(\vec{r}, \vec{\xi})$ and $(\vec{r}, \vec{R})$ is specified by Eq. (3.6) or, to first order, by Eq. (3.9)]. The explicit function $V_{\xi}(\overrightarrow{\mathrm{r}} ; \vec{\xi})$ can be obtained by reexpressing $V(\vec{r} ; \vec{R})$ in terms of the new variables $(\vec{r}, \vec{\xi})$. The same remarks apply to $\sigma$.

Scalar products of the functions $\psi_{n}$ are defined by integration over $\overrightarrow{\mathbf{r}}$ on the surface of constant $\vec{\xi}$,

$$
\left\langle\psi_{m} \mid \psi_{n}\right\rangle=\int \psi_{m}^{*}(\overrightarrow{\mathrm{r}} ; \vec{\xi}) \psi_{n}(\overrightarrow{\mathrm{r}} ; \vec{\xi}) d^{3} \overrightarrow{\mathrm{r}} .
$$

With this definition of the scalar product, $h$ is a Hermitian operator, and since (4.1) is a Schrödinger equation (admittedly with an unusual kinetic and potential energy), the eigenfunctions $\psi_{n}(\vec{r} ; \vec{\xi})$ form a complete orthogonal set.

We can easily show that these states are exact atomic electronic states in the asymptotic channels. For example, in asymptotic channel $B$,

$$
\begin{aligned}
V \rightarrow V_{0}^{B}\left(\overrightarrow{\mathrm{r}}_{B}\right), \quad \cos \sigma \rightarrow & \cos \sigma_{B}=\left(m_{B} / m\right)^{1 / 2}, \\
(\cos \sigma)\left(m^{-1} \vec{\nabla}_{r}^{2}\right)(\cos \sigma) & =\cos \sigma\left(\vec{\nabla}_{\tilde{\eta}}^{2}\right)_{\xi} \cos \sigma \rightarrow \cos ^{2} \sigma_{B}\left(\vec{\nabla}_{\tilde{\eta}}^{2}\right) \AA_{B} \\
& =\left(\vec{\nabla}_{\tilde{r}_{B}}^{2}\right)_{\mathrm{R}_{B}}=m_{B}^{-1} \vec{\nabla}_{r_{B}}^{2} .
\end{aligned}
$$

[We have used Eq. (3.12) to obtain the last result].

The Hamiltonian (4.2) seems to have an unusual kinetic energy operator, but we note that wherever $\sigma$ is a constant, $\cos ^{2} \sigma$ is just a scaling factor which effectively gives a locally correct electronic reduced mass. The symmetrized form $(\cos \sigma) \vec{\nabla} \overrightarrow{\tilde{\eta}}^{2}(\cos \sigma)$ is used to make $h$ Hermitian even when $\sigma$ is not constant.

A direct solution of the differential equation (4.1) for the eigenstates $\psi_{n}(\vec{r} ; \vec{\xi})$ is neither feasible nor necessary. We can learn all we need to know about them .by expressing them in terms of a suitably defined and more familiar set of functions.

\section{B. Relation of $\psi_{n}$ to Born-Oppenheimer eigenfunctions}

Obviously, we should ask "what relation (if any) exists between these functions $\psi_{n}(\overrightarrow{\mathbf{r}} ; \vec{\xi})$ and the BornOppenheimer eigenfunctions defined in Sec. IID?" Care must be taken in answering this question. $\psi_{n}(\vec{r} ; \vec{\xi})$ are defined on surfaces of fixed $\vec{\xi}$, while the Born-Oppenheimer states $\phi_{n}(\overrightarrow{\mathrm{r}} ; \overrightarrow{\mathrm{R}})$ are defined on surfaces of fixed $\vec{R}$; in other words, if we insist on the relation between $\vec{\xi}$ and $\vec{R}$ provided by the transformation (3.6), there is no simple relation between the two sets of functions.

However, we can take an entirely different point of view. For each and every fixed value of the parameter $\vec{\xi}$, the set $\psi_{n}(\vec{r} ; \vec{\xi})$ is complete and orthonormal in $\overrightarrow{\mathrm{r}}$ space; likewise, for each and every fixed value of (another) parameter $\vec{R}$, the set $\phi_{n}(\overrightarrow{\mathrm{r}} ; \overrightarrow{\mathrm{R}})$ is complete and orthonormal in $\overrightarrow{\mathrm{r}}$. There fore, for each and every fixed pair of arbitrary parameters $\vec{\xi}, \vec{R}$, we may expand $\psi_{m}$,

$$
\psi_{m}(\overrightarrow{\mathrm{r}} ; \vec{\xi})=\sum_{n} c_{n m}(\overrightarrow{\mathrm{R}}, \vec{\xi}) \phi_{n}(\overrightarrow{\mathrm{r}} ; \overrightarrow{\mathrm{R}}),
$$




$$
c_{n m}(\overrightarrow{\mathrm{R}} ; \vec{\xi})=\int \phi_{n}^{*}(\overrightarrow{\mathrm{r}} ; \overrightarrow{\mathrm{R}}) \psi_{m}(\overrightarrow{\mathrm{r}} ; \vec{\xi}) d^{3 \overrightarrow{\mathrm{r}}} \text {. }
$$

Of course these formulas are just statements of the orthonormality and completeness of $\left\{\phi_{n}(\overrightarrow{\mathrm{r}} ; \overrightarrow{\mathrm{R}})\right\}$ for every fixed $R$. Above all, we do not assert here that any relation such as (3.6) or (3.9) exists between the parameters $\vec{\xi}$ and $\vec{R}$.

Now, Eqs. (4.5) are valid for arbitrary values of both the fixed parameters $\vec{\xi}$ and $\vec{R}$, but they are not always useful; for example, there is no point in expanding the set $\left\{\psi_{m}(\vec{r} ; \xi=130)\right\}$ in the set $\phi_{n}(\overrightarrow{\mathrm{r}} ; R=0.1)$. The expansion $i s$ useful if we make the fixed parameter $\vec{R}$ equal numerically to the fixed parameter $\vec{\xi}$; i.e., we choose the particular expansion

$$
\psi_{n}(\overrightarrow{\mathrm{r}} ; \vec{\xi})=\sum_{n} C_{n m}(\overrightarrow{\mathrm{R}}=\vec{\xi}) \phi_{n}(\overrightarrow{\mathrm{r}} ; \overrightarrow{\mathrm{R}}=\vec{\xi}) .
$$

These coefficients can be calculated in firstorder perturbation theory by comparing the defining equations for $\psi_{m}(\overrightarrow{\mathrm{r}} ; \vec{\xi})$ and $\left.\phi_{n}(\overrightarrow{\mathrm{r}} ; \overrightarrow{\mathrm{R}})\right|_{\overrightarrow{\mathrm{R}}=\vec{\xi}}$. These are

$$
\begin{aligned}
h \psi_{n}(\overrightarrow{\mathrm{r}} ; \vec{\xi})= & {\left[-\left(\hbar^{2} / 2 m\right) \cos \sigma_{\xi}(\overrightarrow{\mathrm{r}} ; \vec{\xi})\left(\vec{\nabla}_{r}^{2}\right) \dot{\xi} \cos \sigma_{\xi}(\overrightarrow{\mathrm{r}} ; \vec{\xi})\right.} \\
& \left.+V_{\xi}(\overrightarrow{\mathrm{r}} ; \vec{\xi})\right] \psi_{n}(\overrightarrow{\mathrm{r}} ; \vec{\xi}) \\
= & W_{n}(\vec{\xi}) \psi_{n}(\overrightarrow{\mathrm{r}} ; \vec{\xi})
\end{aligned}
$$

and

$$
\begin{aligned}
{\left[-\left(\hbar^{2} / 2 m\right)\left(\vec{\nabla}_{r}^{2}\right)_{\overrightarrow{\mathrm{R}}}+V(\overrightarrow{\mathrm{r}} ; \overrightarrow{\mathrm{R}})\right]_{\overrightarrow{\mathrm{R}}=\vec{\xi}}\left[\phi_{n}(\overrightarrow{\mathrm{r}} ; \overrightarrow{\mathrm{R}})\right]_{\mathbf{R}=\vec{\xi}} } \\
=\left[\epsilon_{n}(\overrightarrow{\mathrm{R}}) \phi_{n}(\overrightarrow{\mathrm{r}} ; \overrightarrow{\mathrm{R}})\right]_{\overrightarrow{\mathrm{R}}=\vec{\xi}} .
\end{aligned}
$$

To first order, provided the $\phi_{n}^{\prime}$ 's are not degenerate, we have

$$
\begin{aligned}
& W_{n}(\vec{\xi})=\epsilon_{n}(\vec{R}=\vec{\xi}) \\
& +\int \phi_{n}(\overrightarrow{\mathrm{r}} ; \overrightarrow{\mathrm{R}}=\vec{\xi}) h^{\prime} \phi_{n}(\overrightarrow{\mathrm{r}} ; \overrightarrow{\mathrm{R}}=\vec{\xi}) d^{3} \overrightarrow{\mathrm{r}}, \\
& c_{n n}=1 \text {, } \\
& c_{n m}(\overrightarrow{\mathrm{R}}=\vec{\xi})=-\int \frac{\phi_{n}(\overrightarrow{\mathrm{r}} ; \overrightarrow{\mathrm{R}}=\vec{\xi}) h^{\prime} \phi_{m}(\overrightarrow{\mathrm{r}} ; \overrightarrow{\mathrm{R}}=\vec{\xi})}{\left[\epsilon_{n}(\overrightarrow{\mathrm{R}})-\epsilon_{m}(\overrightarrow{\mathrm{R}})\right]_{\vec{R}=\vec{\xi}}} \frac{d^{3} \overrightarrow{\mathrm{r}}}{},
\end{aligned}
$$

where

$$
\begin{aligned}
h^{\prime}= & h-h_{\mathrm{BO}}(\overrightarrow{\mathrm{r}} ; \overrightarrow{\mathrm{R}}=\vec{\xi}) \\
= & -\left(\hbar^{2} / 2 m\right)\left[\cos \sigma_{\xi}(\overrightarrow{\mathrm{r}} ; \vec{\xi})\left(\vec{\nabla}_{r}^{2}\right)_{\xi} \cos \sigma_{\xi}(\overrightarrow{\mathrm{r}} ; \vec{\xi})-\left(\vec{\nabla}_{r}^{2}\right)_{\vec{\xi}}\right] \\
& +V_{\xi}(\overrightarrow{\mathrm{r}} ; \vec{\xi})-V(\overrightarrow{\mathrm{r}} ; \overrightarrow{\mathrm{R}}=\vec{\xi}) .
\end{aligned}
$$

$h^{\prime}$ is easily shown to contain terms of order $(m / \mu)$ and smaller. (cf. Sec. VB).

If the Born-Oppenheimer eigenstates are degenerate then the standard formulas of degenerate perturbation theory give the $\psi$ 's as linear combinations of $\phi$ 's. The most important application of this type occurs in the case of "isotopic mole-

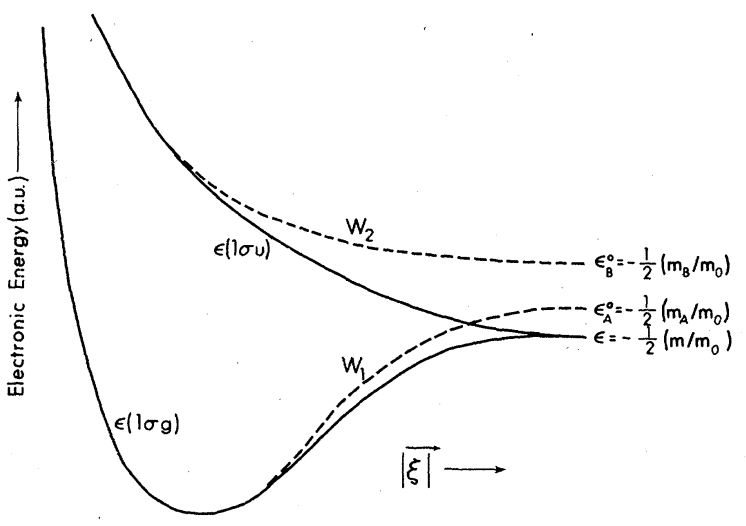

FIG. 7. Qualitative correlation diagram for the BormOppenheimer eigenvalues $\epsilon\left(1 \sigma_{g}\right), \epsilon\left(2 \sigma_{u}\right)$, and the "exact adiabatic" eigenvalues $W_{1}, W_{2}$, for an "isotopic molecule" (electrically symmetric but heteronuclear system). Nuclei of charge +1 . Isotope splittings are exaggerated several thousandfold for clarity.

cules": As $R \rightarrow \infty$, the Born-Oppenheimer states for a symmetric but heteronuclear system are degenerate in $(g, u)$ pairs, while we have shown above that the corresponding states $\psi_{m}(\overrightarrow{\mathrm{r}} ; \vec{\xi})$ become exact atomic electronic states of $A$ and $B$ as $|\vec{\xi}| \rightarrow \infty$. In Sec. VI, we show that first-order perturbation theory within degenerate manifolds of $(g, u)$ Born-Oppenheimer states yields the correct atomic states and isotopic shifts to lowest nonzero order in $m / \mu$.

For the special case of isotopic molecules, there is a significant difference between the properties of $\psi_{m}$ and $W_{m}$ as functions of $|\vec{\xi}|$ and those of $\phi_{n}$ and $\epsilon_{n}$ as functions of $|\vec{R}|=|\vec{\xi}|$ : The states $\psi_{m}$ behave adiabatically with respect to isotope splittings, while the BO states $\phi_{n}$ are diabatic in character; i.e., the isotopic perturbation $h^{\prime}$ is not diagonal in the basis $\phi_{n}(\overrightarrow{\mathrm{r}} ; \overrightarrow{\mathrm{R}}=\vec{\xi})$. Figure 7 depicts these correlations in a qualitative way. While it is nice to define a basis like $\psi_{m}$ which goes smoothly and adiabatically to the isotopically distinct atomic states, it is not really a very useful basis for physical purposes, because nearly all real collisions are so fast that the system behaves diabatically with respect to the isotope splitting $\mathrm{s}^{4 \mathrm{~b}}$ (see the discussion in Sec. VI).

\section{Born-Oppenheimer basis on the $\vec{\xi}$ surface}

Actually, the precise form of the relation between the basis sets $\left\{\psi_{n}(\vec{r} ; \vec{\xi})\right\}$ and $\left\{\phi_{n}(\vec{r} ; \vec{R}=\vec{\xi})\right\}$ is less important than the fact that such a relationship exists. The expansion (4.6) implies that the functions $\left\{\phi_{n}(\overrightarrow{\mathrm{r}} ; \overrightarrow{\mathrm{R}}=\vec{\xi})\right\}$ can be used as a basis set on the $\vec{\xi}$ surface. In effect, the replacement of the fixed parameter $\vec{R}$ by the numerically equal 
fixed parameter $\vec{\xi}$ provides a mapping of the BornOppenheimer basis functions from the surface of constant $\vec{R}$ to the surface of constant $\vec{\xi}$, such that the functions still form a complete and orthonormal set.

Of course when we use this basis set we will find that the isotope shifts are asymptotically offdiagonal, but this is not a serious problem; except at very low energies the isotope shifts are negligible, and when they are important they can be treated by the perturbative methods discussed in Sec. VI.

The dual role of the vectors $\vec{R}$ and $\vec{\xi}$ - as dynamical variables related by Eqs. (3.6) or (3.9), and as fixed parameters which are made numerically equal - can be very confusing if it is not handled with great care. In the next section, we will first transform the Hamiltonian from $(\vec{r}, \vec{R})$ to $(\vec{r}, \vec{\xi})$ coordinates; $\vec{R}$ and $\vec{\xi}$ are then the dynamical variables, related by Eq. (3.9). Afterwards, we will expand the wave function $\Psi(\vec{r}, \vec{\xi})$ in terms of the basis set $\left\{\phi_{n}\right\}$ on the surface of constant $\vec{\xi}$; in that context, $\vec{R}$ and $\vec{\xi}$ are regarded as fixed parameters taken to be numerically equal; either may replace the other.

\section{COUPLED.EQUATIONS OF SCATTERING THEORY}

In this section we obtain coupled differential equations for heavy-particle wave functions, the solution to which provides a fully quantum-mechanical description of slow collisions, correct to order $m / \mu$. If the full wave function is expanded in the basis set $\left\{\psi_{n}(\vec{r} ; \vec{\xi})\right\}$ then the coupled equations (4.16) are obtained. In this representation the scattering problem is well formulated, with exact asymptotic channel eigenstates, and couplings that all vanish asymptotically. However, we have seen that the Born-Oppenheimer basis set $\left\{\phi_{n}(\vec{r} ; \vec{R}=\vec{\xi})\right\}$ is more convenient even though the $\phi_{n}$ are not quite exact atomic eigenstates. In this representation, the coupled equations that result are Eqs. (5.18).

\section{A. Transformation of the Hamiltonian}

We begin by expressing the Hamiltonian in the new dynamical variables $(\vec{r}, \vec{\xi})$. Equations (3.9) specify $\vec{\xi}$ to the required accuracy, and $\vec{s}$ is regarded as a function of $(\vec{r} ; \vec{R})$. Define the $k$ th Cartesian components of new momenta conjugate to $(\overrightarrow{\mathrm{r}}, \vec{\xi})$,

$$
p_{k}=-i \hbar\left(\partial / \partial r_{k}\right)_{\vec{\xi}}, \quad P_{k}=-i \hbar\left(\partial / \partial \xi_{k}\right)_{\overrightarrow{\mathbf{r}}}
$$

and introduce the quantities

$$
\gamma_{k j}=\left(\partial s_{j} / \partial \gamma_{k}\right)_{\vec{R}}
$$

$$
\Gamma_{k j}=\left(\partial s_{j} / \partial R_{k}\right)_{\overrightarrow{\mathbf{r}}} \cdot
$$

Then the old and new momenta are related by the equations (summation over repeated indices is understood):

$$
\begin{aligned}
& \left.\left(-i \hbar \vec{\nabla}_{r}\right)_{k}\right|_{\vec{R}}=p_{k}+(m / 2 \mu)\left(\gamma_{k j} P_{j}+P_{j} \gamma_{k j}\right), \\
& \left.\left(-i \hbar \vec{\nabla}_{R}\right)_{k}\right|_{\overrightarrow{\mathbf{r}}}=P_{k}+(m / 2 \mu)\left(\Gamma_{k j} P_{j}+P_{j} \Gamma_{k j}\right) .
\end{aligned}
$$

Here, as in Eqs. $\left(3.3^{\prime \prime}\right)$ of Ref. 1 we have symmetrized the second term in each of these equations so that the new momenta (as well as the old) are Hermitian operators. Putting Eqs. (5.4) into Eq. (2.3c), we find the new Hamiltonian, correct to order $m / \mu$, is

$$
\begin{aligned}
H=(2 \mu)^{-1} & \left\{P_{k} P_{k}+\frac{1}{2}\left(\gamma_{k j} P_{j}+P_{j} \gamma_{k j}\right) p_{k}+\frac{1}{2} p_{k}\left(\gamma_{k j} P_{j}+P_{j} \gamma_{k j}\right)\right. \\
& +(m / \mu)\left[\frac{1}{4}\left(\gamma_{k j} P_{j}+P_{j} \gamma_{k j}\right)\left(\gamma_{k l} P_{l}+P_{l} \gamma_{k l}\right)\right. \\
& \left.\left.+\frac{1}{2}\left(\Gamma_{k j} P_{j}+P_{j} \Gamma_{k j}\right) P_{k}+\frac{1}{2} P_{k}\left(\Gamma_{k j} P_{j}+P_{j} \Gamma_{k j}\right)\right]\right\} \\
& +(2 m)^{-1} p_{k} p_{k}+V_{\xi}(\overrightarrow{\mathrm{r}} ; \vec{\xi})+O(m / \mu)^{2} .
\end{aligned}
$$

Here, $\gamma_{k j}$ and $\Gamma_{k j}$ are functions of $(\vec{r}, \vec{R})$; however, we can expand them as Taylor series about $\vec{R}^{\prime}=\vec{\xi}$, where, as required by $(3.9), \vec{R}-\vec{R}^{\prime}=-(m / \mu) \overrightarrow{\mathrm{S}}$; then we have

$$
\begin{aligned}
\gamma_{k j}(\overrightarrow{\mathrm{r}} ; \overrightarrow{\mathrm{R}}) & =\left[\partial s_{j}(\overrightarrow{\mathrm{r}} ; \overrightarrow{\mathrm{R}}) / \partial \gamma_{k}\right]_{\overrightarrow{\mathrm{R}}^{\prime}}-(m / \mu)\left(\partial \gamma_{k j} / \partial R_{l}\right) s_{l}+\cdots \\
& =\gamma_{k j}(\overrightarrow{\mathrm{r}} ; \vec{\xi})-(m / \mu)\left(\partial \Gamma_{l j} / \partial \gamma_{k}\right)_{\xi} s_{l}(\overrightarrow{\mathrm{r}} ; \vec{\xi})+\cdots
\end{aligned}
$$

A similar relation holds for $\Gamma_{k j}$. This means that we may consider $\gamma_{k j}$ and $\Gamma_{k j}$ in Eq. (5.5) to be functions of $(\overrightarrow{\mathrm{r}} ; \vec{\xi})$, provided only that we add a small correction to the terms of order $m / \mu$ in the heavy-particle kinetic energy operator (terms in curly brackets). [A more careful examination of the expressions (3.3a) and (3.3b) for the transformed momenta in Ref. 1 will show that just the same corrections should appear there.]

The new Hamiltonian is not as complicated as it looks, and can be much simplified by a further approximation: in addition to neglecting terms of orders $(m / \mu)^{2}$ and higher, we also neglect terms proportional to $\sim(m / \mu) \operatorname{grad} f$, which appear in the heavy-particle kinetic energy. Then the Hamiltonian can be written

$$
\begin{aligned}
H^{\prime}=(2 \mu)^{-1} & \left\{P_{k} P_{k}+\frac{1}{2}\left[\gamma_{k j} P_{j}+P_{j} \gamma_{k j}\right] p_{k}\right. \\
& +\frac{1}{2} p_{k}\left[\gamma_{k j} P_{j}+P_{j} \gamma_{k j}\right] \\
& \left.+(m / \mu)\left[\gamma_{k j} \gamma_{k l}+2 \delta_{k l} \Gamma_{k j}\right] P_{j} P_{l}\right\} \\
& +(2 m)^{-1} p_{k} p_{k}+V_{\xi}(\overrightarrow{\mathrm{r}} ; \vec{\xi})+\cdots .
\end{aligned}
$$

The terms of order $m / \mu$ in the heavy-particle kinetic energy vanish (neglecting terms $\sim(m / \mu) \operatorname{grad} f)$, if we define $\overrightarrow{\mathbf{s}}$ by Eq. (3.9b); if we use $(3.9 \mathrm{c})$, they are given by 


$$
\begin{aligned}
(2 \mu)^{-1}(m / \mu)\left[\gamma_{k j} \gamma_{k l}+2 \Gamma_{k j} \delta_{k l}\right] P_{j} P_{i} \\
\quad=(2 \mu)^{-1}(m / 4 \mu)\left[f^{2}-1\right] P_{k} P_{k} .
\end{aligned}
$$

\section{Channel limit behavior}

The Hamiltonian (5.7) has a very simple meaning anywhere that $f$ is approximately constant. For example, in the $B$ channel, $f=1, \vec{\xi} \rightarrow \overrightarrow{\tilde{R}}_{B}$, and Eq. (5.7) becomes

$$
\begin{array}{r}
-\left(\hbar^{2} / 2 \mu_{B}\right)\left[\vec{\nabla}_{R_{B}}^{2}+\left(\mu_{B} / \mu\right)^{1 / 2}(1+\lambda) \vec{\nabla}_{R_{B}} \cdot \vec{\nabla}_{r}\right] \\
-\left(\hbar^{2} / 2 m\right) \vec{\nabla}_{r}^{2}+V ;
\end{array}
$$

but to within errors $\sim(m / \mu)^{2}$, the kinetic energy in (5.9) is identical to that given by Eq. (2.4a): the $\vec{\nabla}_{R_{B}} \cdot \vec{\nabla}_{r}$ cross term is present simply because $\left(\overrightarrow{\mathrm{R}}_{B}, \overrightarrow{\mathrm{r}}\right)$ do not form "orthogonal" Jacobi coordinates. At finite $R,(5.7)$ contains additional terms proportional to gradients of $f$, which arise from curvature on the surfaces of constant $\vec{\xi}$. The equivalence of (5.9) and (2.4) shows that nothing significant has been omitted from the Hamiltonian (5.7), and we will use that Hamiltonian in the rest of the paper. The terms of order $(m / \mu)$ $\times \operatorname{grad} f$, which have been neglected in (5.7), may of course be retained if desired.

\section{B. Coupled equations in the $\psi_{n}$ representation}

We write the full wave function as an expansion in the complete orthogonal set $\left\{\psi_{n}(\overrightarrow{\mathrm{r}} ; \vec{\xi})\right\}$ defined in Sec. IV A,

$$
\Psi_{\xi}(\vec{r} ; \vec{\xi})=\sum_{n} \chi_{n}(\vec{\xi}) \psi_{n}(\vec{r} ; \vec{\xi}) .
$$

Straightforward calculation using the Hamiltonian (5.7) gives the coupled equation

$$
\begin{aligned}
(2 \mu)^{-1}\{ & {\left[-i \hbar \vec{\nabla}_{\xi}+\underline{\overrightarrow{\mathrm{P}}}(\xi)+\underline{\overrightarrow{\mathrm{A}}}(\xi)\right]^{2} } \\
+ & \left.(m / \mu) \underline{D}(\xi)\left(-i \hbar \vec{\nabla}_{\xi}\right)^{2}\right\} \underline{\underline{\chi}}(\vec{\xi}) \\
& +\left[\underline{W}(\xi)+\underline{\Lambda}(\xi)-(2 \mu)^{-1} \underline{\overrightarrow{\mathrm{A}}} \cdot \underline{\overrightarrow{\mathrm{A}}}\right] \underline{\chi}(\xi)=E \underline{\chi}(\vec{\xi}),
\end{aligned}
$$

where

$$
\overrightarrow{\mathbf{P}}_{m n}(\xi)=\left\langle\psi_{m}\left|\left(-i \hbar \vec{\nabla}_{\xi}\right)\right| \psi_{n}\right\rangle .
$$

$W$ is the diagonal matrix whose elements are $\bar{W}_{n}(\xi)$ [cf. Eqs. (4.1)], and the matrices $\underline{A}, \underline{D}$, and $\Lambda$ are defined as follows:

$$
\overrightarrow{\mathrm{A}}_{m n}(\xi)=-i \hbar\left\langle\psi_{m}\left|\left\{\vec{\nabla}_{r}(\overrightarrow{\mathrm{s}}) \cdot \vec{\nabla}_{r}+\frac{1}{2} \vec{\nabla}_{r}^{2}(\overrightarrow{\mathrm{s}})\right\}\right| \psi_{n}\right\rangle
$$

(i.e., the $j$ th Cartesian component is

$$
A_{m n}^{j}=-i \hbar\left\langle\psi_{m}\left|\left\{\left(\partial s_{j} / \partial r_{k}\right)\left(\partial / \partial r_{k}\right)+\frac{1}{2}\left(\partial^{2} s j / \partial r_{k}^{2}\right)\right\}\right| \psi_{n}\right\rangle \text {. }
$$

If we assume neglect of terms $\sim(m / \mu) \operatorname{grad} f$, the matrix $D$ vanishes for $\overrightarrow{\mathrm{s}}$ given by Eq. $(3.9 \mathrm{~b})$, while if we use $(3.9 \mathrm{c})$,

$$
D_{m n}(\xi)=+\left(\frac{1}{4}\right)\left\langle\psi_{m}\left|\left(f^{2}-1\right)\right| \psi_{n}\right\rangle .
$$

Finally, $\Lambda$ is given by

$$
\Lambda_{m n}=\left\langle\psi_{m}\left|-\left(\hbar^{2} / 2 m\right) \sin ^{2} \sigma \vec{\nabla}_{r}^{2}\right| \psi_{n}\right\rangle,
$$

a result which follows from the definition (4.2) for the electronic Hamiltonian, again dropping terms $\sim(m / \mu) \operatorname{grad} f$; but, using Eq. (3.8), we can write this as

$$
\Lambda_{m n}=-\left(\hbar^{2} / 2 \mu\right)\left\langle\psi_{m}\left|\left[\frac{1}{2}(f+\lambda)\right]^{2} \vec{\nabla}_{r}^{2}\right| \psi_{n}\right\rangle,
$$

and (to within errors $\sim(m / \mu) \operatorname{grad} f$ ) this is just equal to $(2 \mu)^{-1} \overrightarrow{\mathrm{A}} \cdot \overrightarrow{\mathrm{A}}$. These terms thus cancel, and Eqs. (5.11) become

$$
\begin{aligned}
&(2 \mu)^{-1}\left\{\left[-i \hbar \vec{\nabla}_{\xi}+\underline{\overrightarrow{\mathrm{P}}}+\underline{\overrightarrow{\mathrm{A}}}\right]^{2}+\right.\left.(m / \mu) \underline{D}\left(-i \hbar \vec{\nabla}_{\xi}\right)^{2}\right\} \underline{\chi(\xi)} \\
&+\underline{W}(\xi) \underline{\chi}(\vec{\xi})=E \underline{\chi}(\xi) .
\end{aligned}
$$

Let us examine the various terms in Eqs. (5.16). The matrix $\overrightarrow{\mathrm{P}}$ contains the standard "nonadiabatic" couplings, and, insofar as lowest-order nondegenerate perturbation theory is valid [cf. Eqs. (4.9) and (4.10) ] its elements in this representation are the same as those of PSS theory. It is easy to show that some matrix elements of $\overrightarrow{\mathrm{P}}$ do not vanish as $\xi=|\vec{\xi}|-\infty$, and that the matrix $\overrightarrow{\mathrm{A}}$ asymptotically exactly cancels $\overrightarrow{\mathrm{P}}$. For the case of "isotopic molecules," since the diagonal matrix $W$ yields the exact atomic electronic energies, the functions $\psi_{n}$ do not behave like the Born-Oppenheimer states $\phi_{n}(\overrightarrow{\mathrm{r}} ; \overrightarrow{\mathrm{R}}=\bar{\xi})$; instead they behave adiabatically with respect to the isotopic splittings (cf. Fig. 7). In this case, therefore, $\overrightarrow{\mathrm{P}}$ contains additional nonadiabatic couplings (not present in PSS theory) which reflect the mixing of $g$ and $\underline{u}$ molecular states to produce $A$ and $B$ atomic states as $\xi \rightarrow \infty$.

We will not discuss the remaining term $D$ here, except to note that in any case it vanishes asymptotically, since $f^{2}=1$ wherever bound-state electron density is finite, as $\xi \rightarrow \infty$. A brief discussion of this term is given in the more useful Born-Oppenheimer representation (cf. Sec. VC).

This representation of the scattering problem has a formal elegance, since the scattering boundary conditions may be exactly satisfied (to any required accuracy in $m / \mu$ ), and therefore the theory of scattering. which results is entirely free of any formal defects. On the other hand, the electronic basis functions $\psi_{n}$ are complicated; they obey an unfamiliar electronic Schrödinger equation, and, where their properties are qualitatively different from those of the set $\left\{\phi_{n}(\vec{r} ; \vec{\xi})\right\}$, the latter provide a more convenient representation of actual collision behavior (cf. Sec. VI). 
C. Coupled equations in Born-Oppenheimer representation

We have seen that the functions $\left\{\phi_{n}(\vec{r} ; \vec{R})\right\}$ form a complete orthogonal basis on a surface of constant $\vec{\xi}$ provided we employ the replacement $\vec{R}=\vec{\xi}$. Then we expand the full wave function

$$
\Psi(\overrightarrow{\mathrm{r}} ; \vec{\xi})=\sum_{n} \chi_{n}(\vec{\xi}) \phi_{n}(\overrightarrow{\mathrm{r}} ; \vec{\xi})
$$

Now, of course, the $\chi_{n}$ 's are different functions from those in Eq. (5.10), since the basis is different. They obey the coupled equations

$$
\begin{aligned}
& \left\{(2 \mu)^{-1}\left[-i \hbar \vec{\nabla}_{\xi}+\underline{\overrightarrow{\mathrm{P}}}(\xi)+\underline{\overrightarrow{\mathrm{A}}}(\xi)\right]^{2}+\underline{\epsilon}(\xi)\right\} \underline{\chi}(\xi) \\
& \quad+\left\{\underline{\Delta}(\xi)-(2 \mu)^{-1} \underline{\overrightarrow{\mathrm{A}}} \cdot \underline{\overrightarrow{\mathrm{A}}}\right\} \underline{\chi}(\xi) \\
& \quad+(2 \mu)^{-1}(m / \mu) \underline{D}(\xi)\left(-i \hbar \vec{\nabla}_{\xi}\right)^{2} \underline{\chi}(\xi)=E \underline{\chi} \underline{(\xi)} .
\end{aligned}
$$

In these equations, matrix elements are taken between the BO molecular states but otherwise the matrices $\vec{P}, \vec{A}$, and $\vec{D}$ are as defined previously; $\epsilon$ is the diagonal matrix of Born-Oppenheimer $\overline{e l e c t r o n i c}$ eigenvalues. In this representation, $\vec{A}$ may also be written

$$
\overrightarrow{\mathrm{A}}_{m n}(\xi)=(i m / \hbar)\left(\epsilon_{m}-\epsilon_{n}\right)\left\langle\phi_{m}|\vec{s}| \phi_{n}\right\rangle .
$$

Finally the matrix $\Delta$ is defined

$$
\Delta_{m n}=\left\langle\phi_{m}\left|\left[V_{\xi}(\overrightarrow{\mathrm{r}} ; \vec{\xi})-V(\overrightarrow{\mathrm{r}} ; \vec{\xi})\right]\right| \psi_{n}\right\rangle .
$$

Equations (5.18) are identical to those obtained by the ETF formulation [Eqs. (3.7) of Ref. 1] but have been derived rigorously (in these differential equations, since $\vec{\xi}$ is now merely a variable name, we may replace it by the name $\vec{R}$; this is notationally more consistent with its actual role inside $\phi_{n}$ and in matrix elements, and makes the practical relation to PSS theory clear). We have discussed the effect of the ETF corrections $\vec{A}$ on the nonadiabatic couplings in Ref. 1 ; here we turn our attention to the smaller terms in these equations, which are now placed on a firm footing.

\section{Electronic binding energy corrections}

The terms $\left[\Delta-(2 \mu)^{-1} \overrightarrow{\mathrm{A}} \cdot \overrightarrow{\mathrm{A}}\right]$ are corrections, of order $\sim(m / \mu) \bar{\epsilon}$, to the binding energy of the electron, due to differences between the molecular reduced mass $m$ and the corresponding atomic reduced masses $m_{A}, m_{B}$. They reproduce isotopic term splittings in the case of systems like $\mathrm{HD}^{+}$, and should not be neglected unless these effects are deemed unimportant. In $\mathrm{HD}^{+}$they are unimportant for collision energies above $\sim 0.05 \mathrm{eV} .{ }^{15}$

The matrix $\Delta$ may be related to more easily computed or familiar quantities. Keeping in mind the recent notation change, we have, from Eq. (4.3a),

$$
V_{\xi}(\overrightarrow{\mathbf{r}} ; \vec{\xi}=\overrightarrow{\mathrm{R}})=V[\overrightarrow{\mathbf{r}} ; \overrightarrow{\mathbf{R}}-(m / \mu) \overrightarrow{\mathbf{s}}],
$$

and using a Taylor expansion about $R$ we obtain

$$
\Delta_{m n}=-(m / \mu)\left\langle\phi_{m}\left|\left[\overrightarrow{\mathrm{s}} \cdot \vec{\nabla}_{R} V(\overrightarrow{\mathrm{r}} ; \overrightarrow{\mathrm{R}})\right]\right| \phi_{n}\right\rangle ;
$$

symmetrizing and inserting a set of intermediate states, we write

$$
\begin{aligned}
\Delta_{m n}=-\frac{1}{2}(m / \mu) \sum_{l} & \left\{\left\langle\phi_{m}|\overrightarrow{\mathrm{s}}| \phi_{l}\right\rangle\left\langle\phi_{l}\left|\vec{\nabla}_{R} V\right| \phi_{n}\right\rangle\right. \\
+ & \left.\left\langle\phi_{n}\left|\vec{\nabla}_{R} V\right| \phi_{l}\right\rangle\left\langle\phi_{l}|\overrightarrow{\mathrm{s}}| \phi_{n}\right\rangle\right\}
\end{aligned}
$$

and then, noting that $\vec{\nabla}_{R} V=\vec{\nabla}_{R} h_{\mathrm{BO}}$, we use the Hellmann-Feynman theorem,

$$
\left\langle\phi_{l}\left|\vec{\nabla}_{R} h_{\mathrm{BO}}\right| \phi_{n}\right\rangle=\delta_{l n}\left(\vec{\nabla}_{R} \epsilon_{n}\right)+(i / \hbar)\left(\epsilon_{n}-\epsilon_{l}\right) \overrightarrow{\mathrm{P}}_{l n},
$$

and obtain

$$
\begin{aligned}
\Delta_{m n}=-(m / \mu)\{ & \left\{\overrightarrow{\mathrm{s}}_{m n} \cdot \vec{\nabla}_{R}\left[\frac{1}{2}\left(\epsilon_{m}+\epsilon_{n}\right)\right]\right. \\
& +(i / \hbar)\left(\epsilon_{n}-\epsilon_{m}\right)[\underline{\overrightarrow{\mathrm{s}}} \cdot \underline{\overrightarrow{\mathrm{P}}}+\underline{\overrightarrow{\mathrm{P}}} \cdot \overrightarrow{\mathrm{s}}]_{m n} \\
& \left.-(2 \mu)^{-1}[\underline{\overrightarrow{\mathrm{P}}} \cdot \underline{\overrightarrow{\mathrm{A}}}+\underline{\overrightarrow{\mathrm{A}}} \cdot \overrightarrow{\overrightarrow{\mathrm{P}}}]_{m n}\right\} .
\end{aligned}
$$

For a nonsymmetric system, where the BO states $\phi_{n}$ are uniquely correlated to atomic states, it is easy to show that the diagonal elements of $\left[\Delta-(2 \mu)^{-1} \overrightarrow{\mathrm{A}} \cdot \overrightarrow{\mathrm{A}}\right]$ give the atomic energies [correct to $\left.\sim(m / \mu)^{2}\right]$. We have

$$
\Delta_{n n}=-(m / \mu) \overrightarrow{\mathrm{S}}_{n n} \cdot \vec{\nabla}_{R} \epsilon_{n}-(2 \mu)^{-1}[\underline{\overrightarrow{\mathrm{A}}} \cdot \underline{\overrightarrow{\mathrm{P}}}+\underline{\overrightarrow{\mathrm{P}}} \cdot \overrightarrow{\mathrm{A}}]_{n n} ;
$$

asymptotically, $\vec{\nabla}_{R} \epsilon_{n}$ vanishes, $\underline{\overrightarrow{\mathrm{A}}} \longrightarrow-\underline{\overrightarrow{\mathrm{P}}}$, and therefore

$$
\lim _{R \rightarrow \infty}\left[\underline{\Delta}-(2 \mu)^{-1} \overrightarrow{\mathrm{A}} \cdot \overrightarrow{\mathrm{A}}\right]_{n n}=(2 \mu)^{-1}[\underline{\overrightarrow{\mathrm{P}}} \cdot \underline{\overrightarrow{\mathrm{P}}}]_{n n} .
$$

Using the identity

$$
-\hbar^{2}\left\langle\phi_{m}\left|\vec{\nabla}_{R}^{2}\right| \phi_{n}\right\rangle=[\underline{\overrightarrow{\mathrm{P}}} \cdot \underline{\overrightarrow{\mathrm{P}}}]_{m n}-i \hbar \vec{\nabla}_{R} \cdot \overrightarrow{\mathrm{P}}_{n n},
$$

we find, since $\vec{\nabla}_{R} \cdot \underline{\overrightarrow{\mathrm{P}}} \rightarrow 0$ asymptotically, that the diagonal correction is just equal to

$$
\begin{aligned}
-\left(\hbar^{2} / 2 \mu\right) & \left\langle\phi_{n}\left|\vec{\nabla}_{R}^{2}\right| \phi_{n}\right\rangle \\
& =-\left(\hbar^{2} / 2 \mu\right)\left\langle\phi_{n_{J}}\left|\vec{\nabla}_{R}^{2}\right| \phi_{n_{J}}\right\rangle \\
& =-\left(\hbar^{2} / 2 \mu\right)\left[\frac{1}{2}\left(f_{J}+\lambda\right)\right]^{2}\left\langle\phi_{n_{J}}\left|\vec{\nabla}_{r_{J}}^{2}\right| \phi_{n_{J}}\right\rangle \\
& =-\frac{1}{2} \hbar^{2}\left[m_{J}^{-1}-m^{-1}\right]\left\langle\phi_{n_{J}}\left|\vec{\nabla}_{r_{J}}^{2}\right| \phi_{n_{J}}\right\rangle \simeq\left(\epsilon_{n_{J}}^{0}-\epsilon_{n_{J}}\right),
\end{aligned}
$$

(a result proved in Ref. 1 , Sec. IIC 2).

For the more important symmetric case, the molecular states $\phi_{n}$ are asymptotically degenerate in $(g, u)$ pairs and the isotopic correction term must be evaluated within the $2 \times 2$ degenerate subspaces; then using degenerate perturbation theory it can be shown that (to order $m / \mu$ ) the correct atomic energy levels and zero-order atomic states are recovered (cf. Sec. VI). 
Finally we note that in general the off-diagonal matrix elements of $\left[\Delta-(2 \mu)^{-1} \overrightarrow{\mathrm{A}} \cdot \overrightarrow{\mathrm{A}}\right]$ also do not vanish asymptotically between nondegenerate levels $(m, n)$. These infinite-range couplings necessarily result because the Born-Oppenheimer states and the exact atomic states do not quite agree (since they have different électronic reduced masses). However, these couplings are exceedingly tiny, we know precisely what they mean, and they are of no importance to collision problems.

\section{Transport kinetic energy corrections}

The effects represented by the matrix $D$ depend on the choice made for the form of $\overrightarrow{\mathrm{s}}$ [Eq $\overrightarrow{\mathrm{s}}$. (3.9b and $3.9 \mathrm{c})]$. In arriving at the simple expression (5.14) [for choice (3.9c)] or $D \equiv 0$ [for (3.9b)], we also have neglected terms of order $\sim(m / \mu) \operatorname{grad} f$; if such terms are retained, then either choice for $\overrightarrow{\mathrm{s}}$ leads to nonzero corrections of this type. In writing this expression, we also have kept only those terms in which $\left(-i \hbar \vec{\nabla}_{R}\right)$ acts upon $\chi(\vec{R})$; this yields a quantity of the order of the heavyparticle momentum, while (as shown in Ref. 1) the effect of this operator upon $\phi_{n}$ is to yield quantities of the order of the electronic momentum (the discarded terms add corrections to the nonadiabatic coupling $\sim(m / \mu)(\overrightarrow{\mathbf{P}}+\overrightarrow{\mathbf{A}})$.

The diagonal elements of the matrix $D$ have a simple interpretation; they represent transient corrections (nonzero only for finite $R$ ) to the heavy-particle kinetic energy, $\sim(m / \mu) E$.

Off-diagonal elements of $D$ can in principle lead to inelastic scattering. However, they lead to interactions proportional to the square of the heavy-particle speed $v_{N}$. Using the rough estimate $\left|(\overrightarrow{\mathrm{P}}+\overrightarrow{\mathrm{A}})_{m n}\right| \sim p_{e} \simeq m v_{e}$ where $v_{e}$ is the orbital electron speed, we see that the ratio of the $D$-type interactions to the nonadiabatic interactions arising from $\overrightarrow{\mathrm{P}}+\overrightarrow{\mathrm{A}}$ is $\sim\left(v_{N} / v_{e}\right)$. For slow collisions this quantity is assumed to be small, but it is clear that for increasing collision energies the effects of the $\underline{D}$ terms must be considered.

\section{CHARGE EXCHANGE IN THE HD ${ }^{+}$SYSTEM}

We now consider specifically the "isotopic molecule" problem described in the Introduction as a critical test of the adequacy of the theory. We will prove first that the spurious $(g, u) \overrightarrow{\mathrm{P}}$-matrix couplings of PSS theory [see Eq. $(\overline{1}, \overline{2})]$ are exactly canceled by the corresponding $\vec{A}$-matrix elements. Then we shall show that the real couplings in the system arise from the isotope splitting terms $\Delta-(2 \mu)^{-1} \overrightarrow{\mathrm{A}} \cdot \overrightarrow{\mathrm{A}}$ within asymptotically degenerate manifolds of $\overline{(g}, u)$ molecular states.
A. Removal of spurious PSS couplings

In Ref. 1, Sec. IV A 5, we showed that all terms arising directly from the mass asymmetry $\lambda$ in PSS theory are exactly cancelled by corresponding terms in the ETF correction matrix A. In particular, from Eqs. (4.4), (4.5) of Ref. 1, we find that

$$
\underline{\overrightarrow{\mathrm{P}}}+\underline{\overrightarrow{\mathrm{A}}}=\underline{\overrightarrow{\mathrm{P}}}_{g}+\underline{\overrightarrow{\mathrm{A}}}_{g},
$$

and that, since $\left[-i \hbar \vec{\nabla}_{R}\right]_{\vec{r}_{g}}$ and $\overrightarrow{\mathrm{S}}_{g}$ have gerade parity, the right-hand side of Eq. (6.1) cannot connect states of different parities. Therefore no nonadiabatic couplings exist within the relevant asymptotically degenerate pairs of molecular states.

\section{B. Isotopic splittings}

For a symmetric system, let $\phi_{n_{g}}$ and $\phi_{n_{u}}$ be an asymptotically degenerate pair of $\mathrm{BO}$ states.

These are asymptotically related to approximate states $\phi_{n_{A}}, \phi_{n_{B}}$ :

$\phi_{n_{g}}=2^{-1 / 2}\left(\phi_{n_{B}}+\phi_{n_{A}}\right), \quad \phi_{n_{u}}=2^{-1 / 2}\left(\phi_{n_{B}}-\phi_{n_{A}}\right)$.

Now, as shown in Eqs. (5.26) and (5.27), we know the asymptotic elements of the quantity

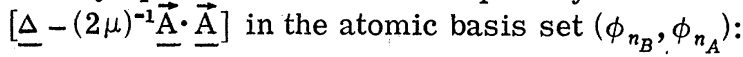

$$
\begin{aligned}
& \left\langle\phi_{n_{B}}\left|-\left(\hbar^{2} / 2 \mu\right) \vec{\nabla}_{R}^{2}\right| \phi_{n_{B}}\right\rangle \\
& =-\frac{1}{2} \hbar^{2}\left(m_{B}^{-1}-m^{-1}\right)\left\langle\phi_{n_{B}}\left|\vec{\nabla}_{r_{B}}^{2}\right| \phi_{n_{B}}\right\rangle, \\
& \left\langle\phi_{n_{A}}\left|-\left(\hbar^{2} / 2 \mu\right) \vec{\nabla}_{R}^{2}\right| \phi_{n_{A}}\right\rangle \\
& =-\frac{1}{2} \hbar^{2}\left(m_{A}^{-1}-m^{-1}\right)\left\langle\phi_{n_{A}}\left|\vec{\nabla}_{r_{A}}^{2}\right| \phi_{n_{A}}\right\rangle, \\
& \left\langle\phi_{n_{B}}\left|-\left(\hbar^{2} / 2 \mu\right) \vec{\nabla}_{R}^{2}\right| \phi_{n_{A}}\right\rangle=0 ;
\end{aligned}
$$

and of course, from this we may infer via (6.2) its matrix elements in the $(g, u)$ representation,

$$
\begin{aligned}
\left\langle\phi_{n_{g}}\left|-\left(\hbar^{2} / 2 \mu\right) \vec{\nabla}_{R}^{2}\right| \phi_{n_{g}}\right\rangle & \\
& =\left\langle\phi_{n_{u}}\left|-\left(\hbar^{2} / 2 \mu\right) \vec{\nabla}_{R}^{2}\right| \phi_{n_{u}}\right\rangle \\
& =-\frac{1}{2} \hbar^{2}\left[\frac{1}{2}\left(m_{B}^{-1}+m_{A}^{-1}\right)-m^{-1}\right]\left\langle\phi_{n_{B}}\left|\vec{\nabla}_{r_{B}}^{2}\right| \phi_{n_{B}}\right\rangle,
\end{aligned}
$$

and

$$
\begin{aligned}
\left\langle\phi_{n_{g}}\right|- & \left(\hbar^{2} / 2 \mu\right) \vec{\nabla}_{R}^{2}\left|\phi_{n_{u}}\right\rangle \\
& =-\frac{1}{2} \hbar^{2}\left[\frac{1}{2}\left(m_{B}^{-1}-m_{A}^{-1}\right)\right]\left\langle\phi_{n_{B}}\left|\vec{\nabla}_{r_{B}}^{2}\right| \phi_{n_{B}}\right\rangle,
\end{aligned}
$$

as well as the obvious fact that making it diagonal again recovers the correct zero-order atomic states $\phi_{n_{B}}, \phi_{n_{A}}$ and the first-order isotopic energy shifts, as given by (6.3).

\section{Treatment of scattering problem}

In the Born-Oppenheimer (molecular state) representation, the nondiagonal matrix $\left[\Delta-(2 \mu)^{-1} \overrightarrow{\mathrm{A}} \cdot \overrightarrow{\mathrm{A}}\right]$, whose asymptotic elements are given by Eqs. (6.4), provides couplings between 
the states $\phi_{n_{g}}, \phi_{n_{u^{*}}}$ This is a diabatic representation. To solve the problem in this representation:

(i) Integrate the coupled equations $(5.18)$, including the isotopic splitting terms, for the twostate $(g, u)$ manifold $\left(1 s \sigma_{g}, 2 p \sigma_{u}\right)$ from the origin out to some suitably large but finite $R=R_{0}$.

(ii) Transform to the atomic representation (basis states $\phi_{1 s_{A}}, \phi_{1 s_{B}}$ ) and continue the integration to convergence of the $S$-matrix calculation. In this representation, $\left[\Delta-(2 \mu)^{-1} \vec{A} \cdot \vec{A}\right]$ is diagonal, but now $h_{\mathrm{BO}}$ is not diagonal.

(iii) A good matching point $R_{0}$ is the value of $R$ such that $\left|\epsilon_{1 s \sigma_{g}}-\epsilon_{2 p \sigma_{u}}\right|_{R_{0}} \simeq$ (isotopic splitting). For $\mathrm{HD}^{+}$this is about 11.8 a.u.

Alternatively, had we wished we could have described the same problem using the states $\psi_{n}(\overrightarrow{\mathrm{r}} ; \overrightarrow{\mathrm{R}})$, and solving Eqs. (5.16). In this basis, we know $W$ is a diagonal matrix and the states $\psi_{n}$ each go to correct atomic states asymptotically, while internally, as shown in Sec. IV A, they closely resemble the Born-Oppenheimer states $\phi_{n}$. This is an adiabatic representation; in the region where the Born-Oppenheimer splitting $\left(\epsilon_{g}-\epsilon_{n}\right)$ and the isotope splitting $\left(\epsilon_{1 s_{A}}^{0}-\epsilon_{1 s_{B}}^{0}\right)$ are of comparable magnitude, the states $\psi_{n}$ undergo a change of character from molecular to atomic states as $R$ increases. In this region, there is a nonadiabatic coupling $\overrightarrow{\mathbf{P}}$ which links the two states, and solution of Eqs. (5.16) with this coupling included will also describe the isotopic charge exchange.

However, description of the problem in the representation based on $\psi_{n}$ is not really a good idea. In the first place, the $\psi_{n}$ 's and the $W_{n}$ 's are not easy to compute, nor are the nonadiabatic couplings $\overrightarrow{\mathrm{P}}$, but in addition, the actual behavior during collision is really not adiabatic; it is more nearly diabatic, and in that case the Born-Oppenheimer basis states, and the solution technique described above using them, are more appropriate.

Detailed calculations on charge exchange in $\mathrm{HD}^{+}$ at very low energies have recently been completed by Davis and Thorson. ${ }^{15}$

\section{DISCUSSION}

Let us now review the main ideas in this work, to see which are essential and which are secondary and could have been developed differently. First of all, what really is wrong with the usual PSS theory? Useful perspective on this question is provided by Figs. 4 and 6: We have to solve the Schrödinger equation for a particle moving on an $n$-dimensional surface. Direct numerical solution in $n$ dimensions is impractical, and we attempt to take advantage of the approximate separability of some degrees of freedom (e.g., "elec- tronic motion") from others. This is done by expanding the full wave function in a discrete set of basis functions defined on some $j$-dimensional surface in the full configuration space; the Schrödinger equation thereby is reduced to a set of coupled differential equations in the remaining $N_{-j} j$ dimensions, hopefully a more tractable form. For this purpose it is always mathematically possible to use any $j$-dimensional surface and any set of functions defined on that surface; as long as the expansion basis is complete, and boundary conditions are expressed properly, then the exact solution to the full set of coupled equations is the solution to the full Schrödinger equation. But expansion bases are never actually complete; in real life they must be truncated to a small, very incomplete set. The physical problem, then (as opposed to the purely mathematical one) is to choose an appropriate surface, and an appropriate set of functions on that surface, such that the separation of degrees of freedom is as good as possible and even with a truncated basis set will still yield an accurate solution to the full Schrödinger equation.

In the usual formulation of PSS theory, the surface chosen is a surface of constant $\vec{R}$, and the basis functions are the Born-Oppenheimer states $\left\{\phi_{n}(\overrightarrow{\mathrm{r}} ; \overrightarrow{\mathrm{R}})\right\}$. If the expansion included the mathematically complete set of these states, then the PSS theory could be exact. However, as was emphasized by Thorson and his co-workers, ${ }^{14}$ that complete set would have to include substantial contributions from the continuum states in the BornOppenheimer representation, rendering the coupled equations quite intractable. In this paper and that preceding it we have described the problems that arise with a truncated expansion in the PSS representation: (i) infinite-range couplings $(\overrightarrow{\mathrm{P}} \neq 0)$ as $R \rightarrow 0$; (ii) unrealistically large cross sections for direct impact processes such as ionization ${ }^{14}$; (iii) large, unphysical couplings between $g$ and $u$ electronic states in isotopic molecules. In the PSS theory, either the surface, or the expansion basis, or both, is not quite good enough.

The essential idea of the preceding pape ${ }^{1}$ is to modify the basis set of Born-Oppenheimer functions to include the electron translation factors, while still keeping the surfaces of constant $\vec{R}$ as those on which the expansion is defined. A truncated basis set can then provide an accurate representation, given certain assumptions and approximations. In retrospect, we can see now that the minor difficulties and ambiguities of the ETF approach really arise because we have not quite obtained a good separation of "degrees of freedom"; we then have to include "translation" effects in the "electronic" wave functions.

The essential idea of the present paper we owe 
to Mittleman ${ }^{2}$ : in effect, he proposed that $a$ different surface of definition for the basis functions can provide a better representation than that of PSS theory. Figures 4 and 6 make it quite clear why this is so: By defining the "scattering coordinate" $\vec{\xi}$, and defining expansion bases on surfaces of constant $\vec{\xi}$, we achieve a much better separation of "translational" and "electronic" degrees of freedom. The true translational motion is "in the $\vec{\xi}$ direction," not "the $\vec{R}$ direction"; once this is recognized, and the internal states defined on the surface normal to that direction, all need for electron translation factors goes away and the proper physical couplings between different degrees of freedom follow directly, and easily.

Mittleman's definition of the coordinate $\vec{\xi}$ is not quite the correct one. In our notation, he defines it

$$
\vec{\xi}_{M}=\overrightarrow{\mathrm{R}}+(m / \mu)\left\{\frac{1}{2}[f+\lambda]\left[\overrightarrow{\mathrm{r}}-\frac{1}{2}(f+\lambda) \overrightarrow{\mathrm{R}}\right]\right\}+O(m / \mu)^{2},
$$

which differs from our definition $(3.9 b)$ by a factor of 2 in the last part of the $m / \mu$ term. Several arguments can be given to show that the definitions (3.9) are correct rather than (7.1).

The definitions (3.9) follow naturally from the transformation relations (3.3) connecting mass scaled coordinates for the channel and molecular regions. Mittleman's definition of $\vec{\xi}$ gives the correct unscaled channel coordinates $\overrightarrow{\mathrm{R}}_{A}$ and $\overrightarrow{\mathrm{R}}_{B}$, but takes no account of the reduced masses $\mu_{A}, \mu_{B}$. But the idea of separation of internal and translational degrees of freedom and the idea that the latter motion is normal to the surface of definition of the former is clearly evident only in the mass-scaled coordinates; in unscaled coordinates the transformations corresponding to (3.3) are not orthogonal. Indeed the main purpose of mass scaled coordinates in the entire theory is to create the simple picture displayed in Figs. 4 and 6 . If we use Mittleman's definition for $\vec{\xi}$, we find that although the spurious PSS coupling (1.2) is removed, an equally paradoxical asymmetric $g-u$ coupling, Eq. (1.5), appears instead; this only disappears when the differences between $\mu_{A}, \mu_{B}$, and $\mu$ are taken into account.

Another way of describing this point is to compare Figs. 4 and 6 . For the symmetric homonuclear case (Fig. 4), surfaces of constant $\vec{\xi}$ are distorted relative to surfaces of constant $\vec{R}$; for the symmetric but heteronuclear case $\lambda \neq 0$ (Fig. 6), they are not only distorted, but also rotated by an angle $\sigma_{0}$ defined in Eq. $(3.13 \mathrm{c})$. It is not hard to show that the rotation by itself completely eliminates the fictitious $g, u$ couplings. Then the distortion superimposed on this rotation provides a coordinate system which asymptotically agrees with the atomic channel coordinates and thereby accounts for the motion of the electron as it is carried along with the nuclei. In order to introduce no further $(g, u)$ couplings, it is only essential that the distortion be described by gerade contributions to $\vec{\xi}$. The definitions (3.9) both have these properties; the ungerade part is just the term that would arise from rotation by $\sigma_{0}$, and the gevade part is a distortion that makes $\vec{\xi}$ go to $\overrightarrow{\tilde{\mathrm{R}}}_{A}$ or $\overrightarrow{\overrightarrow{\mathrm{R}}}_{B}$ in the channels.

The two definitions of $\vec{\xi}$ considered in these papers are not the only ones having the required rotation and distortion characteristics; a trivial class of modifications is obtained by scaling with any desired constant, for example, and there are other more complicated choices as well. For the two definitions specified in (3.9b) and (3.9c), surfaces of constant $\vec{\xi}$ have slightly different curvatures and distortions at finite $\xi$, but are identical in the channel limits. Either choice gives rise to a formally acceptable theory, and at present we have no convincing arguments in support of one over the other. The two definitions give rise to somewhat different results for the ETF correction matrix $\overrightarrow{\mathrm{A}}$; in the case of $(3.9 \mathrm{~b})$ the element $\overrightarrow{\mathrm{A}}_{m n}$ has an additional term

$$
\overrightarrow{\mathrm{A}}_{m n}^{f^{2}}=(i m / \hbar) \frac{1}{8} \overrightarrow{\mathrm{R}}\left\langle\phi_{m}\left|\left(1-f^{2}\right)\right| \phi_{n}\right\rangle,
$$

which does not appear in the case of (3.9c). We have computed some of these terms for transitions in the $\mathrm{H}_{2}{ }^{+}$and $\mathrm{HeH}^{++}$systems and we find that they are often of significant size. If we use Eq. (3.9c) instead, these terms do not appear at all, and the only price paid for this is a change in the much smaller terms associated with the matrix $D$. Further study will be needed, however, before we can say which choice may be more useful. The problem posed by this flexibility in defining $\vec{\xi}$ is thus similar to that discussed in Ref. 1 regarding the choice of the switching function $f(\vec{r} ; \vec{R})$. The really critical step in the theory, however, is the definition of some appropriate scattering coordinate $\vec{\xi}$, and the decision to define electronic basis functions on a surface of constant $\vec{\xi}$; other aspects of the formalism are matters of practical and physical sense, and a variety of alternatives is open.

For example, our choice (3.11) for the electron coordinate $\vec{\eta}$ is only one of many possibilities, such as (3.12) or (3.13) which we discussed earlier. It does not matter what coordinate system we use on the surface of fixed $\vec{\xi}$, provided the mapping $(\vec{r} ; \vec{R})$ to $(\vec{\eta}, \vec{\xi})$ is one-to-one. We chose $\vec{\eta}=\vec{r}$ because the transformed Hamiltonian (5.7) then has the simplest form for the general asymmetric heteronuclear system.

Similarly, great freedom exists in the choice of 
basis functions. It is most convenient, of course, if they form an orthogonal set, since the coupled equations are simpler, but the orthogonality may be defined in many ways. We can use a set that is orthogonal with any (reasonable) positive definite weighting factor $\rho(\vec{\eta})$

$$
\int \psi_{m}^{*}(\vec{\eta} ; \vec{\xi}) \psi_{n}(\vec{\eta} ; \vec{\xi}) \rho(\vec{\eta}) d^{3} \vec{\eta}=\delta_{m n} \cdot
$$

We chose $\rho=1$, but other choices are equally valid; Mittleman and Tai, ${ }^{2}$ for example, chose $\rho$ to be equal to the Jacobian of the $(\vec{r}, \vec{R}) \rightarrow(\vec{\eta}, \vec{\xi})$ transformation (this differs from unity by terms $\sim m / \mu$ ). However, such a choice produces some additional complications we have avoided here.

The basis functions form a complete set if they are eigenfunctions of an "electronic Hamiltonian" which is Hermitian with respect to the chosen weight factor $\rho$. We have developed the theory using such complete sets, but it should be recognized that a well chosen set of "pseudostates" can give a very accurate description of the scattering regardless of their formal completeness properties.

Of the two basis sets discussed here, the set $\left\{\psi_{n}(\vec{r} ; \vec{\xi})\right\}$ has mainly formal merits; these states go continuously and adiabatically to exact atomic eigenstates in the channels, so that in this representation the scattering theory is very well formulated with no infinite-range couplings. But this basis is not particularly useful for actual calculations, as we showed in Secs. IV and V.

For general utility, probably no basis set can compete with the set of Born-Oppenheimer functions $\left\{\phi_{n}(\vec{r} ; \vec{\xi})\right\}$ defined on the surface of constant $\vec{\xi}$. They do not quite become exact atomic channel eigenstates, but the small isotopic couplings which remain are easily included (when relevant) by the methods of Sec. VI.

In this discussion we have not raised the question of diabatic or partially diabatic representations, which really has no direct connection with the main ideas here. It is clear that in addition to the set $\left\{\phi_{n}\right\}$, we could use any set obtained from it by $\vec{\xi}$-dependent unitary transformations. Of course the matrix elements must be correspondingly modified; in particular, Eqs. (5.19), (5.21), (5.22), etc., are true only in the Born-Oppenheimer basis, and the diagonal matrix $\underline{\epsilon}$ must be replaced by the (nondiagonal) matrix $\underline{h}_{B 0^{\circ}}$.

\section{CONCLUSION}

The coupled equations (A3) obtained from the usual perturbed stationary states (PSS) formulation of slow collision theory are incorrect, and lead to serious physical errors for the analysis of many processes. These equations should be modified to include the additional terms due to the matrices $\vec{A}, \Delta$, and $D$, as in Eqs. (5.18). The most important change is the replacement of the PSS "nonadiabatic coupling" matrix $\overrightarrow{\mathrm{P}}$ by $\overrightarrow{\mathrm{P}}+\overrightarrow{\mathrm{A}}$; the effects of this modification have been discussed at length in the preceding paper. ${ }^{1}$ The cor rection $\overrightarrow{\mathrm{A}}$ removes spurious contributions to $\overrightarrow{\mathrm{P}}$ both in the collision region and asymptotically. The terms $\Delta-(2 \mu)^{-1} \overrightarrow{\mathrm{A}} \cdot \overrightarrow{\mathrm{A}}$ give the asymptotic isotope shifts, and in addition there are some small terms $\sim(m / \mu) E$ associated with the matrix $D$, whose diagonal elements yield a correction to the "transport kinetic energy" of the electron, while the off-diagonal couplings are negligible in comparison to the effects of nonadiabatic couplings $\underline{\vec{P}}+\vec{A}$ in the low-velocity limit. Derivations of the correct coupled equations (5.18) have been given by two independent formulations, here and in the preceding paper and a unified physical interpretation has been given to these coupled equations. Calculations of these new terms and their effects on various processes are currently in progress.

\section{ACKNOWLEDGMENT}

This work has been supported in part by the Research Corporation (U.S.A.), the NSF, NASA, and the National Research Council of Canada.

\section{APPENDIX: A PARADOX OF PSS THEORY}

Here we show that for isotopic molecules like $\mathrm{HD}^{+}$, the naive application of the PSS theory does not agree with the classical impact parameter theory. In a physically sensible formulation of the impact parameter theory, there is no $(\underline{g}, \underline{u})$ coupling; in the PSS theory, though, there is a large $(\underline{g}, \underline{u})$ coupling which does not vanish in the classical limit.

\section{A. Impact parameter theory}

In this method it is assumed $a$ priori that the nuclei move on a (rectilinear) classical path; the electron then sees a time-dependent force field and obeys the time-dependent Schrödinger equation

$$
h_{\mathrm{IP}}(\vec{\rho} ; t) \Upsilon(\vec{\rho} ; t)=i \hbar \frac{\partial}{\partial t}[\Upsilon(\vec{\rho} ; t)],
$$

where

$$
h_{\mathrm{IP}}(\vec{\rho} ; t)=-\left(\hbar^{2} / 2 m\right) \vec{\nabla}_{r}^{2}+V(\vec{\rho} ; t)
$$

and for the moment we do not raise the question of the reference origin for the electron coordinate $\vec{\rho}$.

Note the following points: (a) The masses of the nuclei nowhere appear in this formulation; the 
collision is that of two infinitely massive potential fields moving past each other, with an electron moving between them. (b) Since the nuclei are infinitely massive, there can be no distinction between the rest mass of the electron and its atomic or molecular reduced masses $\left(m_{0}=m_{A}=m_{B}=m\right)$. (c) Regardless of the nuclear masses, if the system is symmetric, then the potential $V(\vec{\rho} ; t)$ is invariant with respect to inversion about the geometric center of the system, $\overrightarrow{\mathrm{r}}_{g} \rightarrow-\overrightarrow{\mathrm{r}}_{g}$; it follows that if the reference origin for the electron coordinates be taken as the geometric center, $\vec{\rho}=\overrightarrow{\mathrm{r}}_{g}$, then electronic parity is exactly conserved and there is rigorously no coupling between $g$ and $\underline{u}$ states. This is obvious on physical grounds a $\overline{l o n e}$ for the $\mathrm{H}_{2}{ }^{+}$system, and the crux of the argument is that-at this level of approximation-the equations for $\mathrm{HD}^{+}$are identical to those for $\mathrm{H}_{2}{ }^{+}$, since the potential is the same.

\section{B. PSS theory}

Figure 1 shows the molecular coordinate system for an isotopic molecule; the electron coordinate $\vec{r}$ has its origin at the center of mass of the nuclei, and the kinematic Hamiltonian is given by Eq. (2.3c). We expand $\Psi(\vec{r} ; \vec{R})$ in a complete discrete set of electronic states,

$$
\Psi(\overrightarrow{\mathbf{r}} ; \overrightarrow{\mathrm{R}})=\sum_{n} \chi_{n}(\overrightarrow{\mathrm{R}}) \Phi_{n}(\overrightarrow{\mathrm{r}} ; \overrightarrow{\mathrm{R}})
$$

and obtain the coupled equations

$$
\left[(1 / 2 \mu)\left(-i \hbar \vec{\nabla}_{R}+\underline{\mathrm{P}}^{2}+\underline{h}(\overrightarrow{\mathrm{R}})\right] \underline{\chi}(\overrightarrow{\mathrm{R}})=E \chi(\overrightarrow{\mathrm{R}}) .\right.
$$

Since the electronic Hamiltonian $h$ is invariant under parity transformation it can have no matrix elements liking $\underline{g}$ and $\underline{u}$ states. However, the matrix $\overrightarrow{\mathrm{P}}$ has nonvanishing and large $(\underline{g}, \underline{u})$ matrix elements,

$$
\begin{aligned}
\overrightarrow{\mathrm{P}}_{g u} & =\left\langle g\left|\left(-i \hbar \vec{\nabla}_{R}\right)_{r}\right| u\right\rangle \\
& =\left\langle g\left|\left[\left(-i \hbar \vec{\nabla}_{R}\right)_{\overrightarrow{\mathrm{r}}_{g}}-\frac{1}{2} \lambda\left(-i \hbar \vec{\nabla}_{r}\right)_{\overrightarrow{\mathrm{R}}}\right]\right| u\right\rangle \\
& =-\frac{1}{2} \lambda\left\langle g\left|\left(-i \hbar \vec{\nabla}_{r}\right)_{\overrightarrow{\mathrm{R}}}\right| u\right\rangle, \\
& =-\frac{1}{2} \lambda(i m / \hbar)\left(\epsilon_{g}-\epsilon_{n}\right)\left\langle g\left|\overrightarrow{\mathrm{r}}_{g}\right| u\right\rangle .
\end{aligned}
$$

This matrix element vanishes within the manifold of a degenerate $(g, u)$ pair as $R \rightarrow \infty$, but it has finite values at finite $R$; for $\mathrm{HD}^{+}$it is about $0.1 \hbar / a_{0}$ as $R \rightarrow 0$. Note further that it does not depend on the atomic isotopic level splittings, which are proportional to $m_{A}-m_{B}$; instead it is proportional directly to $\lambda=\left(M_{A}-M_{B}\right) /\left(M_{A}+M_{B}\right)$ which remains finite in the limit $M_{A}, M_{B} \rightarrow \infty, m_{0}$ fixed.

\section{Classical limit of PSS theory}

These $(g, u)$ couplings of the PSS equations (A3) do not vanish in the classical limit. The definition of this limit given by Riley ${ }^{5}$ is convenient here: We should obtain the impact parameter equations by taking the limit of large mass at fixed nuclear velocity. To do this, we write

$$
\underline{\chi}(\overrightarrow{\mathrm{R}})=\exp [i \mu \vec{v} \cdot \overrightarrow{\mathrm{R}} / \hbar] \underline{a}(\overrightarrow{\mathrm{R}})
$$

where $\vec{v}$ is a constant velocity in the $Z$ direction with magnitude such that $E=\frac{1}{2} \mu v^{2}$. Equations (A3) then become

$$
\left[(1 / 2 \mu)\left(\mu \vec{v}+\underline{\overrightarrow{\mathrm{P}}}-i \hbar \vec{\nabla}_{R}\right)^{2}+\underline{h}\right] \underline{a}=E \underline{a} .
$$

Collecting terms in powers of $\mu$, as $\mu \rightarrow \infty$ we find

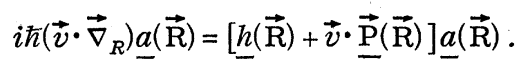

Since $\vec{v} \cdot \vec{\nabla}_{R}=d / d t$, Eqs. (A7) have the same form as do Eqs. (A1) when $\Upsilon$ is there expanded in the set $\left\{\phi_{n}[\overrightarrow{\mathrm{r}} ; \overrightarrow{\mathrm{R}}(t)]\right\}$. However, we showed that Eqs. (A1) can have no $g-u$ couplings, while here in (A7) the couplings $\overrightarrow{\mathrm{P}}_{g u}$ remain. This paradox is only resolved by recognizing that the PSS matrix $\underline{\vec{P}}$ does not give the correct nonadiabatic couplings.

Of course, the argument might be made that, since the impact parameter formulation is properly obtained only as the classical limit of a quantum-mechanical theory, ${ }^{5}$ the reference origin for the electron is not arbitrary but must be prescribed from the quantum-mechanical problem. We have seen that if PSS theory is taken as the quantum-mechanical formulation then this origin is naturally the center of mass of the nuclei. However, this argument does not resolve the paradox; it can be shown that the physical description of the system in the impact parameter limit must be independent of the reference origin, ${ }^{5}$ but now we have two different systems $\left(\mathrm{H}_{2}{ }^{+}, \mathrm{HD}^{+}\right)$whose electronic Hamiltonians $h_{\mathrm{IP}}\left(\overrightarrow{\mathrm{r}}_{\mathrm{g}} ; t\right)$ are identical, and contain no data about nuclear masses, yet hypothetically exhibit different physical behavior in the classical limit, merely because of a difference in choice of reference origin.
${ }^{1}$ W. R. Thorson and J. B. Delos, preceding paper, Phys. Rev. A 17, 117 (1978).

${ }^{2}$ M. H. Mittleman, Phys. Rev. 188, 221 (1969); M. H. Mittleman and H. Tai, Phys. $\overline{R e v}$. A $\underline{8}, 1880$ (1973); M. H. Mittleman, ibid. 9 , 704 (1974); ibid. 10, 442 (1974); J. Chem. Phys. (to be published).
${ }^{3}$ W. R. Gentry (private communication); D. E. Nitz, K. A. Smith, M. W. Geis, R. D. Rundel, and R. F. Stebbings, Bull. Am. Phys. Soc. 21, 1261 (1976), and private communication.

${ }^{4}$ (a) M. Kuriyan, Ph.D. thesis (York University, Toronto, 1974) (unpublished); G. Hunter and M. Kuriyan, in 
Electronic and Atomic Collisions, Abstracts of the Ninth International Conference on the Physics of Electronic and Atomic Collisions, edited by J. S. Risley and R. Geballe (University of Washington, Seattle, 1975), p. 1025. Compare also G. Hunter, B. F. Gray, and H. O. Pritchard, J. Chem. Phys. 45, 3806 (1966); G. Hunter and M. Kuriyan, Proc. R. Soc. A 341,491 (1975); J. Comput. Phys. 21, 319 (1976); G. Hunter, J. Chem. Phys. 64,3213 (1976). (b) A related isoisotopic branching problem in the photodissociation of excited HD has been analyzed by W. R. Thorson [J. Mol. Spectrosc. 37, 199 (1971)].

${ }^{5}$ M. E. Riley, Phys. Rev. A 7, 626 (1973).

${ }^{6} \mathrm{M}$. H. Mittleman (private communication).

${ }^{7} \mathrm{As}$ will be seen later, these Jacobi coordinates form orthogonal systems in six dimensions when appropriately mass scaled.

${ }^{8}$ R. G. Newton, Scattering Theory of Waves and Particles (McGraw-Hill, New York, 1966); N. F. Mott and H. S. W. Massey, Theory of Atomic Collisions, 3rd ed. (Cambridge University, Cambridge, 1965).

${ }^{9}$ They are called "isometric coordinates" by L. A. Pars, in $A$ Treatise on Analytical Dynamics (Heine- man, London, 1965), p. 537.

${ }^{10} \mathrm{~S}$. Glasstone, K. J. Laidler, and H. Eyring [Theory of Rate Processes, 1st ed. (McGraw-Hill, New York,

1941), p. $100 \mathrm{ff]}$ suggest these were first used by J. O. Hirschfelder to study collinear atom-diatom collisions.

${ }^{11}$ E. P. Wigner and L. Eisenbud, Phys. Rev. 72, 29 (1947); A. M. Lane and R. G. Thomas, Rev. Mod. Phys. 30,257 (1958).

${ }^{12}$ Compare for example, A. Kuppermann, Potential Energy Surfaces in Chemistry, edited by W. Lester (IBM Research Laboratories, San Jose, 1971), A. Askar, Bull. Am. Phys. Soc. 21, 1262 (1976).

${ }^{13} \mathrm{R}$. A. Marcus, J. Chem. Phys. $4 \overline{5}, 4493$ (1966); G. Miller and J. C. Light, ibid. $\overrightarrow{54}, 1635$ (1971); R. P. Saxon and J. C. Light, ibid. 56, 3874, 3885 (1972).

${ }^{14}$ W. R. Thorson and H. Levy, II, Phys. Rev. 181, 230 (1969); H. Levy, II and W. R. Thorson, ibid. 181, 244 (1969); 181, 252 (1969); C. F. Lebeda, W. R. Thorson, and H. Levy, II, Phys. Rev. A $\underline{4}, 900$ (1971), Eq. (1); V. SethuRaman, W. R. Thorson, and C. F. Lebeda, ibid. 8,1316 (1973).

${ }^{15} \mathrm{~J}$. P. Davis and W. R. Thorson, Can. J. Phys. (to be published). 